\title{
Orbital spaces in the divide-expand-consolidate coupled cluster method
}

Patrick Ettenhuber, Pablo Baudin, Thomas Kjærgaard, Poul Jørgensen, and Kasper Kristensen

Citation: The Journal of Chemical Physics 144, 164116 (2016); doi: 10.1063/1.4947019

View online: http://dx.doi.org/10.1063/1.4947019

View Table of Contents: http://aip.scitation.org/toc/jcp/144/16

Published by the American Institute of Physics

\section{Articles you may be interested in}

The molecular gradient using the divide-expand-consolidate resolution of the identity second-order Møller-

Plesset perturbation theory: The DEC-RI-MP2 gradient

The Journal of Chemical Physics 145, 024106 (2016); 10.1063/1.4956454

Analytic energy derivatives for the calculation of the first-order molecular properties using the domain-based local pair-natural orbital coupled-cluster theory

The Journal of Chemical Physics 145, 114101 (2016); 10.1063/1.4962369

Sparse maps-A systematic infrastructure for reduced-scaling electronic structure methods. II. Linear scaling domain based pair natural orbital coupled cluster theory

The Journal of Chemical Physics 144, 024109 (2016); 10.1063/1.4939030

Efficient linear-scaling second-order Møller-Plesset perturbation theory: The divide-expand-consolidate RIMP2 model

The Journal of Chemical Physics 144, 054102 (2016); 10.1063/1.4940732

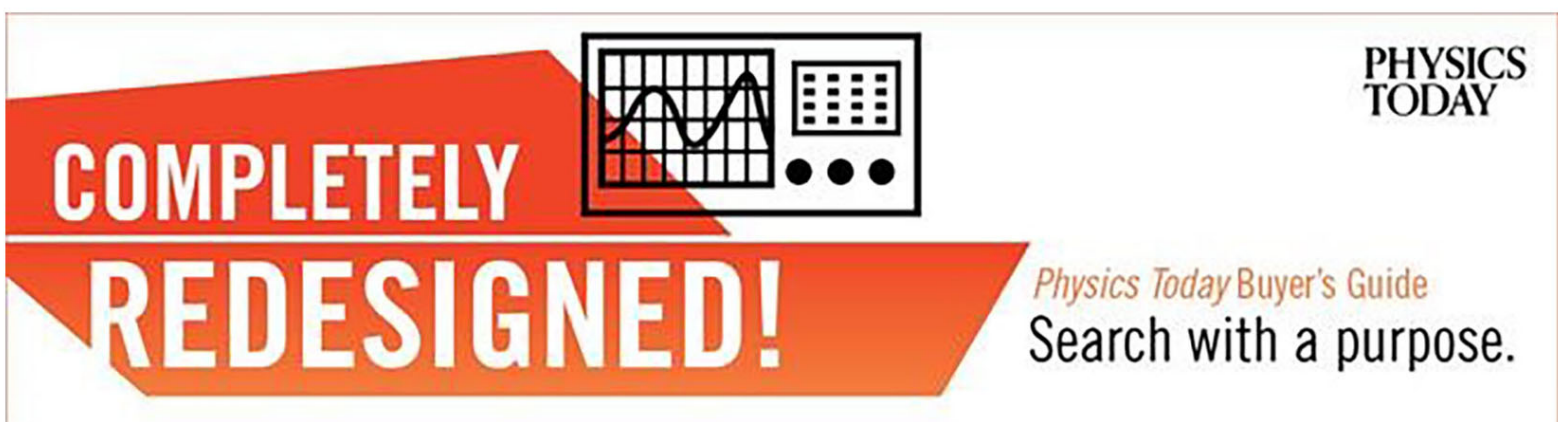




\title{
Orbital spaces in the divide-expand-consolidate coupled cluster method
}

\author{
Patrick Ettenhuber, ${ }^{\text {a) }}$ Pablo Baudin, Thomas Kjærgaard, Poul Jørgensen, \\ and Kasper Kristensen \\ qLEAP Center for Theoretical Chemistry, Department of Chemistry, Aarhus University, \\ Langelandsgade 140, DK-8000 Aarhus C, Denmark
}

(Received 5 February 2016; accepted 5 April 2016; published online 28 April 2016)

\begin{abstract}
The theoretical foundation for solving coupled cluster singles and doubles (CCSD) amplitude equations to a desired precision in terms of independent fragment calculations using restricted local orbital spaces is reinvestigated with focus on the individual error sources. Four different error sources are identified theoretically and numerically and it is demonstrated that, for practical purposes, local orbital spaces for CCSD calculations can be identified from calculations at the MP2 level. The development establishes a solid theoretical foundation for local CCSD calculations for the independent fragments, and thus for divide-expand-consolidate coupled cluster calculations for large molecular systems with rigorous error control. Based on this theoretical foundation, we have developed an algorithm for determining the orbital spaces needed for obtaining the single fragment energies to a requested precision and numerically demonstrated the robustness and precision of this algorithm. Published by AIP Publishing. [http://dx.doi.org/10.1063/1.4947019]
\end{abstract}

\section{INTRODUCTION}

Highly accurate calculations of molecular energies and properties have been feasible for many years using coupled cluster (CC) theory. While it is possible to achieve accuracies challenging experiment, ${ }^{1}$ the $\mathrm{CC}$ hierarchy of methods in a conventional formulation is restricted to small molecules due to an inherent high-polynomial scaling. With the increasing experimental possibilities and general interest in life and material sciences to address more and more complex chemical systems, the need for highly accurate electronic structure calculations for large molecules is growing rapidly. Since the steep scaling of the $\mathrm{CC}$ methods is a direct consequence of the nonlocal nature of the routinely used canonical HartreeFock (HF) orbitals, it was realized early that local orbitals would allow for an accurate linear-scaling description of the electronic correlation effects in large molecules and several local CC methods have been developed since.

Local correlation methods were pioneered by Pulay and Saebo, ${ }^{2,3}$ and an early prominent contribution is the local coupled cluster (LCC) method of Werner and coworkers. ${ }^{4,5}$ Many other local CC methods have been proposed, e.g., the atomic orbital (AO)-based $\mathrm{CC},{ }^{6,7}$ the natural linear scaling approach ${ }^{8}$ the cluster-in-a-molecule approach, ${ }^{9-11}$ the divide-and-conquer approach, ${ }^{12,13}$ the fragment molecular orbital (MO) approach, ${ }^{14,15}$ and the incremental scheme. ${ }^{16,17}$ Linear scaling has also been achieved in second order Møller-Plesset perturbation theory (MP2) calculations using a Laplace-transformation of the energy denominator ${ }^{18}$ with an effective integral screening. ${ }^{19,20}$ In recent years the highly successful pair natural orbitals ${ }^{21-23}$ and orbital specific virtual orbitals $^{24}$ have been used in order to achieve linear scaling

\footnotetext{
a)Electronic mail: pettenhuber@ gmail.com
}

for CC methods. In local CC methods, ad hoc approximations have often been introduced, for example, by assigning fixed virtual correlating orbital spaces to local occupied orbitals, or by a physical fragmentation of the molecule. The precision of a local correlated calculation compared to a standard calculation is in general made unclear by these approximations.

In the standard CC methods, the precision of a calculation is determined by the residual norm threshold for the cluster amplitude equations. The precision is thus defined prior to a calculation. This a priori knowledge of the precision is an important feature and one of the reasons for the success of CC calculations on small molecular systems. In the recently introduced divide-expand-consolidate (DEC) local CC method, ${ }^{25-32}$ the precision is similarly defined prior to a calculation. In the DEC method, a calculation on a full molecular system is divided into a sequence of single fragment and pair fragment calculations referencing small parts of the orbital space of the full molecular system and the precision is defined by the fragment optimization threshold (FOT) that is imposed on the single fragment energy calculations. The FOT is used to identify the orbital spaces where the cluster amplitude equations have to be solved to give the single fragment energies to the FOT precision. Using unions of single fragment orbital spaces in the pair fragment calculations, the precision of the total energy is ultimately determined by the FOT value as demonstrated numerically in Refs. 25-27, 31, and 32. In this paper, we describe how these orbital spaces for the single fragment calculations may be determined for the MP2 and CC with singles and doubles excitations (CCSD) models. The screening of pair fragments that is necessary to obtain a linear-scaling algorithm for determining the total correlation energy with rigorous error control will be described in a forthcoming publication.

A DEC calculation may be described both in terms of an occupied and a virtual partitioning of the $\mathrm{CC}$ correlation 
energy, ${ }^{27}$ which provide two alternative ways of partitioning the correlation energy for the full molecular system into fragment calculations using subsets of the complete molecular orbital space. These two partitioning schemes therefore provide an internal consistency check of the precision ${ }^{29}$ and both schemes have been required for calculating molecular gradients. ${ }^{28}$ We have previously carried out a locality analysis for the occupied partitioning scheme, which demonstrates how orbital spaces may be selected for evaluating the single fragment energies. ${ }^{26}$ In this paper, we improve and generalize this locality analysis to include the virtual partitioning scheme. We analyze the general coupling mechanisms in the MP2 and CCSD amplitude equations theoretically as well as numerically, and we investigate the resulting errors when the amplitude equations are solved in a restricted local orbital space. For MP2, the only coupling mechanism is caused by short-ranged Fock matrix terms in the MP2 amplitude equations, while for the CCSD model, orbital space extensions may also occur via a mechanism involving longrange interactions originating from two-electron integrals in the CCSD amplitude equations. We demonstrate that both the MP2 and CCSD equations may be solved in local restricted orbital spaces with rigorous error control in the single fragment energy.

In Section II we summarize the DEC algorithm. In Section III we analyze the locality of the MP2 and CCSD amplitude equations and identify the coupling mechanisms in the amplitude equations. In Section IV we use this information to develop a practical black-box algorithm that may be used to determine the single fragment energies to the FOT precision. In Section $V$ we analyze the efficiency of the single fragment optimization algorithm numerically and in Section VI we give some concluding remarks.

\section{THE DEC ALGORITHM SUMMARIZED}

In this section we briefly summarize the DEC-CC method with emphasis on giving the background that is required for determining the local orbital spaces needed to solve cluster amplitude equations and obtain the single fragment energies to the predefined FOT tolerance.

The CC correlation energy for a closed-shell molecular system may be expressed as

$$
\begin{aligned}
E_{c o r r} & =\sum_{i a j b}\left(t_{i j}^{a b}+t_{i}^{a} t_{j}^{b}\right)\left(2 g_{i a j b}-g_{i b j a}\right) \\
& =\sum_{i a j b} \tau_{i j}^{a b} L_{i a j b},
\end{aligned}
$$

where $t_{i}^{a}$ and $t_{i j}^{a b}$ are the singles and doubles cluster amplitudes, respectively, $g_{i a j b}$ are two-electron integrals in the Mulliken notation, and we have introduced $\tau_{i j}^{a b}=t_{i j}^{a b}+t_{i}^{a} t_{j}^{b}$ and $L_{i a j b}=2 g_{i a j b}-g_{i b j a}$. Indices $i, j(a, b)$ refer to occupied (unoccupied) HF molecular orbitals (MOs) $\{\phi\}$ and indices $p, q, r, s, t$ denote MO indices of unspecified occupation. For simplicity we assume real MOs throughout the paper.

For a set of local HF orbitals, each orbital may be assigned to a site (e.g., an orbital, a collection of neighboring orbitals, an atom, or a collection thereof) $P, Q, \ldots$ Each site $P$ thus gets assigned a set of occupied $\underline{P}$ and unoccupied $\bar{P}$ orbital indices. For local orbitals a charge distribution $\omega_{p q}=\phi_{p} \phi_{q}$ is nonzero only if the MOs $\phi_{p}$ and $\phi_{q}$ are close in space. An integral $g_{i a j b}$ is thus non-negligible only if the charge distributions $\omega_{i a}$ and $\omega_{j b}$ are non-negligible, i.e., orbitals $\phi_{i}$ and $\phi_{a}$ are assigned to sites that are close to each other and similarly for orbitals $\phi_{j}$ and $\phi_{b}$. If $\phi_{i}$ and $\phi_{j}\left(\phi_{a}\right.$ and $\left.\phi_{b}\right)$ are both assigned to site $P$, we will write $i, j \in \underline{P}(a, b \in \bar{P})$, and $g_{i a j b}$ is non-negligible and contributes to the energy only if $a, b \in[\bar{P}](i, j \in[\underline{P}])$, where the bracket denotes the set of orbitals spatially close to site $P$, including $P$ itself. For compactness we will collectively refer to the local occupied $[\underline{P}]$ and virtual $[\bar{P}]$ orbital spaces as $[P]$.

Replacing the summations in Eq. (1) by summations over sites and pairs of sites and using that some of the two-electron integrals may be neglected (to a given precision), we may write the correlation energy as ${ }^{25,27}$

$$
E_{\text {corr }}^{x}=\sum_{P}\left[E_{P}^{x}+\sum_{Q<P} E_{P Q}^{x}\right],
$$

where $x$ refers to either the occupied $(x=o)$ or the virtual $(x=v)$ partitioning scheme. The occupied single fragment energy $E_{P}^{o}$ for site $P$ and occupied pair interaction energy $E_{P Q}^{o}$ between sites $P$ and $Q$ may be expressed as

$$
E_{P}^{o}=\sum_{i, j \in \underline{P}} \sum_{a, b \in[\bar{P}]} \tau_{i j}^{a b} L_{i a j b}
$$

and

$$
E_{P Q}^{o}=\left(\sum_{\substack{i \in \underline{P} \\ j \in \underline{\underline{Q}}}}+\sum_{\substack{j \in \underline{P} \\ i \in \underline{\underline{Q}}}} \sum_{a, b \in[\bar{P}] \cup[\bar{Q}]} \tau_{i j}^{a b} L_{i a j b} .\right.
$$

The virtual single fragment energy $E_{P}^{v}$ and pair interaction energy $E_{P Q}^{v}$ may similarly be calculated according to

$$
E_{P}^{v}=\sum_{a, b \in \bar{P}} \sum_{i, j \in[\underline{P}]} \tau_{i j}^{a b} L_{i a j b}
$$

and

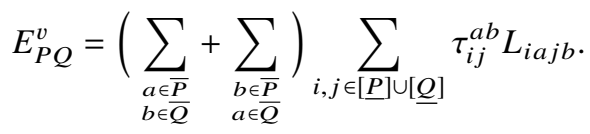

If all orbitals are included in $[\underline{P}]$ and $[\bar{P}]$ for all sites $P$ then Eqs. (1) and (2) are identical for both the occupied and the virtual partitioning schemes.

The evaluation of the occupied single fragment energy $E_{P}^{o}$ requires the amplitudes of the occupied energy orbital space (EOS) in Eq. (3). To indicate that a four-index quantity, $X_{i j}^{a b}$, is restricted to the occupied EOS, we write

$$
X_{i j}^{a b} \in \mathcal{P}_{\mathrm{EOS}}^{o},
$$

where $\mathcal{P}_{\mathrm{EOS}}^{o}$ refers to a collection of four orbital indices with $i, j \in \underline{P}$ and $a, b \in[\bar{P}]$. We will use the following compact notation for $\mathcal{P}_{\text {EOS }}^{o}$ :

$$
\mathcal{P}_{\mathrm{EOS}}^{o} \equiv \underline{P} \times \underline{P} \times[\bar{P}] \times[\bar{P}]=\underline{P}^{2} \times[\bar{P}]^{2} .
$$

Similarly, $E_{P}^{v}$ requires the amplitudes of the virtual EOS from Eq. (5), which can be written as

$$
\mathcal{P}_{\mathrm{EOS}}^{v} \equiv[\underline{P}]^{2} \times \bar{P}^{2} .
$$




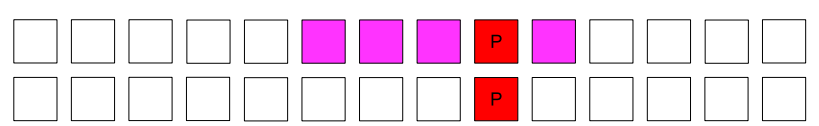

(a)

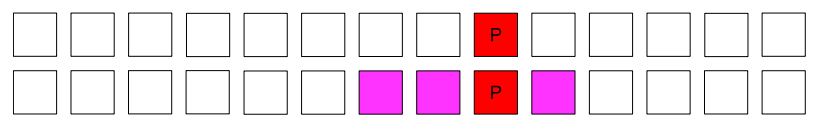

(b)

FIG. 1. Schematic illustration where the upper and lower rows of squares symbolize the sets of virtual and occupied orbitals assigned to a center, respectively. The orbitals assigned to site $P$ are shown as red squares. The space outside $P$ where the integrals $L_{i a j b}$ entering the fragment energy $E_{P}^{x}$ are non-negligible is shown in magenta for the occupied (a) and virtual (b) partitioning schemes. (a) Schematic drawing of $\mathcal{P}_{\text {EOS }}^{o}$. (b) Schematic drawing of $\mathcal{P}_{\text {EOS }}^{v}$

$\mathcal{P}_{\text {EOS }}^{o}$ and $\mathcal{P}_{\text {EOS }}^{v}$ are depicted schematically in Fig. 1. We henceforth use the generic $\mathcal{P}_{\mathrm{EOS}}^{x}, E_{P}^{x}$, and $E_{P Q}^{x}$ to refer to one of the partitioning schemes. In order to obtain the single fragment and pair interaction energies to the predefined precision, the amplitudes of $\mathcal{P}_{\text {EOS }}^{x}$ have to be known, and the accurate determination of these is the focus of the following discussion.

When evaluating fragment energies $E_{P}^{x}$ in local orbital spaces, two primary error types arise. To illustrate these two error types, we consider the evaluation of a fragment energy $E_{P}^{o}$ of the occupied partitioning scheme. The two error types may be summarized as follows:

1. the summation restriction $a b \in[\bar{P}]$ in Eq. (3) in the evaluation of $E_{P}^{o}$;

2. the amplitudes used for evaluating $E_{P}^{o}$ are obtained by solving the amplitude equations in a restricted orbital space.

Note that in the limit where the amplitudes are obtained from a calculation that includes the total orbital space, only error type 1 is present. To schematically illustrate how the two error types affect the size of the orbital space, we have plotted the errors in $E_{P}^{o}$ in Fig. 2 for a typical system. The errors in

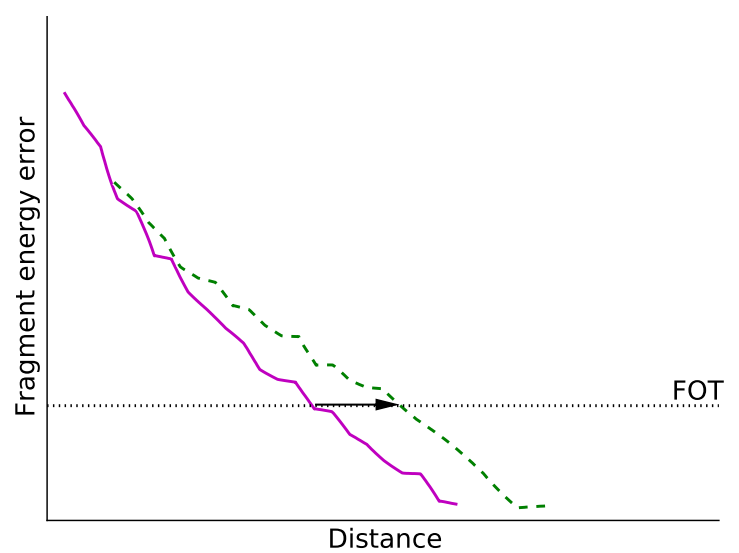

FIG. 2. Schematic illustration of the different error types that are present when fragment energies $E_{P}^{o}$ are evaluated. Each point represents the fragment energy error when orbitals within a certain distance cutoff from site $P$ are included. Magenta, solid line: error type 1; green, dashed line: error types 1 and 2; black arrow: error type 2. See text for details.
$E_{P}^{o}$ are then given for two cases where the orbital space is truncated at a given distance from site $P$. For the solid magenta line, amplitudes have been obtained from a calculation that includes the total molecular orbital space and the summation (Eq. (3)) has been truncated. The magenta line therefore only displays the decay of errors of type 1. For the green dashed line, amplitudes are used where the amplitude equations have been solved in an orbital space that has been truncated at the same distance from $P$ as used for the EOS. Thus, the dashed line contains errors of both types 1 and 2 . The difference between the dashed and solid lines therefore represents errors of type 2. In order to evaluate $E_{P}^{o}$ to the requested FOT precision in a practical calculation, it is therefore necessary to include the coupling space, denoted by the arrow, in addition to the EOS. The mechanisms which define the coupling space in MP2 and CCSD calculations are identified in Section III.

\section{THEORETICAL ANALYSIS: ORBITAL SPACES IN DEC-CC FRAGMENT ENERGY CALCULATIONS}

In this section we carry out a theoretical locality analysis to identify the mechanisms that are responsible for introducing the coupling between the amplitudes of the EOS and the amplitudes of the neighboring orbital spaces. After these mechanisms have been established, we discuss how the coupling may be included in a fragment energy calculation to obtain EOS amplitudes giving the single fragment energy to a predefined precision. In Sections III A and III B we describe the MP2 and CCSD models, respectively, while Section III C contains numerical support for the theoretical analysis. Note that the following discussion is a theoretical analysis, while the practical implementation is described in Section IV.

\section{A. Space extensions in MP2 fragment calculations}

The MP2 amplitude equations constitute a set of linear equations with a positive definite coefficient matrix

$\sum_{c} t_{i j}^{c b} F_{a c}+\sum_{c} t_{i j}^{a c} F_{b c}-\sum_{k} t_{k j}^{a b} F_{k i}-\sum_{k} t_{i k}^{a b} F_{k j}=-g_{a i b j}$.

If a canonical HF basis is used, the Fock matrix is diagonal and Eq. (10) is solved in one iteration. If a basis of local orbitals is used, the Fock matrix is diagonally dominant ${ }^{33}$ and Eq. (10) may be solved in a few iterations using standard iterative algorithms, such as the conjugate gradient or the conjugate residual methods. ${ }^{34,35}$ In this section, we will use the conjugate residual method as an analysis tool for solving the MP2 amplitude equations in a restricted orbital space. For the locality analysis we assume that Eq. (10) is expressed in terms of a set of local HF orbitals and that the Fock matrix and the two-electron integrals are local. These assumptions are backed up numerically in Appendix A for a set of molecules.

\section{Iterative solution of the MP2 amplitude equations}

When solving Eq. (10) using the conjugate residual algorithm, the amplitudes of iteration $(n+1)$ are determined from the amplitudes of iteration $n$, 


$$
t_{i j, n+1}^{a b}=t_{i j, n}^{a b}+\alpha R_{i j, n}^{a b},
$$

where the residual $R_{i j, n}^{a b}$ of iteration $n$

$$
\begin{aligned}
R_{i j, n}^{a b}= & -g_{a i b j}-\sum_{c} t_{i j, n}^{c b} F_{a c}-\sum_{c} t_{i j, n}^{a c} F_{b c} \\
& +\sum_{k} t_{k j, n}^{a b} F_{k i}+\sum_{k} t_{i k, n}^{a b} F_{k j}
\end{aligned}
$$

is used as a search direction. In the conjugate residual method, a line search is performed along the residual direction by optimizing the $\alpha$ parameter. The line search affects the convergence rate of the algorithm, but it does not influence how new orbital spaces are introduced in the iterative algorithm. For simplicity, we therefore set $\alpha=1$ in the rest of this analysis. is

In iteration $n$, the single fragment energy for fragment $P$

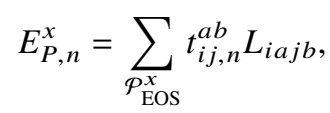

where the summation over $\mathcal{P}_{\text {EOS }}^{x}$ indicates a summation with $t_{i j}^{a b}, L_{i a j b} \in \mathcal{P}_{\text {EOS }}^{x}$ as defined by Eqs. (8) and (9), i.e., for the occupied partitioning scheme $(x=o)$, the indices will be restricted as $i, j \in P$ and $a, b \in[\bar{P}]$, while, for the virtual partitioning scheme $(\bar{x}=v), i, j \in[P]$ and $a, b \in \bar{P}$. Using Eqs. (11) and (13) the energy change in iteration $n, \Delta E_{P, n}^{x}$, is given by

$$
\begin{aligned}
\Delta E_{P, n}^{x} & =E_{P, n}^{x}-E_{P, n-1}^{x} \\
& =\sum_{\mathcal{P}_{\mathrm{EOS}}^{x}} R_{i j, n-1}^{a b} L_{i a j b},
\end{aligned}
$$

where $E_{P, 0}^{x} \equiv 0$. The converged single fragment energy may then be written as

$$
E_{P}^{x}=\sum_{n=1}^{n_{i t}} \Delta E_{P, n}^{x},
$$

where we have used Eqs. (13) and (14) and where $E_{P}^{x}=E_{P, n_{i t}}^{x}$ and $n_{i t}$ is the total number of iterations in the iterative scheme. The residual $R_{n} \in \mathcal{P}_{\text {EOS }}^{x}$ approaches zero with increasing $n$ and $\Delta E_{P, n}^{x}$ therefore becomes negligible. In Sec. III A 2, we will apply the iterative procedure defined by Eqs. (11) and (12) and analyse the propagation of the orbital space throughout the iterative procedure to identify the target orbital space required for determining $E_{P}^{x}$ to the predefined precision.

\section{Propagation of orbital spaces during the iterative procedure}

The EOS amplitudes are defined in the $\mathcal{P}_{\text {EOS }}^{o}$ and $\mathcal{P}_{\text {EOS }}^{v}$ spaces for the occupied and virtual partitioning schemes, respectively, see Fig. 1. However, it is convenient to extend these spaces slightly to put the locality analysis for the occupied and virtual partitioning schemes on an equal footing. We therefore introduce the target space, $\mathcal{P}_{1}$,

$$
\mathcal{P}_{1}=[\underline{P}]^{2} \times[\bar{P}]^{2},
$$

which includes the EOS for both the occupied and virtual partitioning schemes, $\left(\mathcal{P}_{\text {EOS }}^{o} \cup \mathcal{P}_{\text {EOS }}^{v}\right) \subset \mathcal{P}_{1}$.
In the first iteration of the iterative procedure described in Eqs. (11) and (12), all amplitudes are set to zero,

$$
t_{i j, 1}^{a b}=0,
$$

and therefore $E_{P, 1}^{x}=\Delta E_{P, 1}^{x}=0$. The single fragment energy is defined by the EOS amplitudes, and we therefore restrict the residual of the first iteration to the target space $\mathcal{P}_{1}$. Using Eq. (12), we thus obtain

$$
R_{i j, 1}^{a b}=-g_{a i b j} \in \mathcal{P}_{1} .
$$

Using Eqs. (11) and (17), the amplitudes of the second iteration become

$$
t_{i j, 2}^{a b}=R_{i j, 1}^{a b} \in \mathcal{P}_{1} .
$$

The energy difference between the first and second iterations is thus

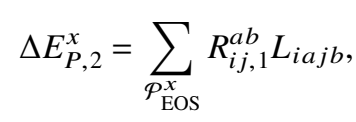

where we have used Eq. (14). The EOS amplitudes in Eq. (19) relax through the iterations and we now identify the coupling mechanism leading to this relaxation.

In the second iteration, the amplitudes in $\mathcal{P}_{1}$ couple directly to sites in the proximity of $[P]$ through summations with non-negligible Fock matrix elements in the residual of Eq. (12). The total (extended) coupling environment of the EOS amplitudes in iteration 2 is denoted $[P]_{2}\left([P] \subset[P]_{2}\right)$. For example, the following terms of Eq. (12) for the second iteration illustrate space extensions of the occupied and virtual spaces, respectively,

$$
\begin{array}{ll}
\sum_{k \in[\underline{P}]} t_{i k, 2}^{a b} F_{k j} & t_{i k, 2}^{a b} \in \mathcal{P}_{1}, \quad j \in[\underline{P}]_{2}, \\
\sum_{c \in[\bar{P}]} t_{i j, 2}^{c b} F_{a c} & t_{i j, 2}^{c b} \in \mathcal{P}_{1}, \quad a \in[\bar{P}]_{2},
\end{array}
$$

where we have used Eq. (19) to restrict the summations in $k$ and $c$. The residual of the second iteration is therefore extended to the space

$$
\mathcal{P}_{2}=[\underline{P}]_{2}^{2} \times[\bar{P}]_{2}^{2},
$$

which is illustrated by Fig. 3 and may be written as

$$
\begin{aligned}
R_{i j, 2}^{a b}= & -g_{a i b j}-\sum_{c} t_{i j, 2}^{c b} F_{a c}-\sum_{c} t_{i j, 2}^{a c} F_{b c}+\sum_{k} t_{k j, 2}^{a b} F_{k i} \\
& +\sum_{k} t_{i k, 2}^{a b} F_{k j} ; \quad R_{i j, 2}^{a b} \in \mathcal{P}_{2}, t_{i j, 2}^{a b} \in \mathcal{P}_{1} .
\end{aligned}
$$

For the following analysis, it is convenient to generalize the notation of Eqs. (16) and (22) and introduce the space $\mathcal{P}_{n}$,

$$
\mathcal{P}_{n}=[\underline{P}]_{n}^{2} \times[\bar{P}]_{n}^{2},
$$

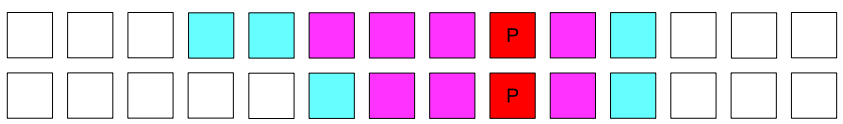

FIG. 3. Iteration 2: The $\mathcal{P}_{1}$ space is symbolized by the squares in red and magenta. The residual is generated in the $\mathcal{P}_{2}$ space (all colored boxes) where the cyan squares symbolize the space extension $\mathcal{P}_{2} \backslash \mathcal{P}_{1}$ in iteration 2 . This space interacts directly with the orbitals of the target space $\mathcal{P}_{1}$. 
where $[\underline{P}]_{n}\left([\bar{P}]_{n}\right)$ is the set of occupied (virtual) orbitals which have non-negligible Fock matrix elements with at least one of the orbitals in $[\underline{P}]_{n-1}\left([\bar{P}]_{n-1}\right)$ and where $[\underline{P}]_{1} \equiv[\underline{P}]$ $\left([\bar{P}]_{1} \equiv[\bar{P}]\right)$. We also note that $\mathcal{P}_{n-1} \subset \mathcal{P}_{n}$ and we will denote the space components of $\mathcal{P}_{n}$ which are not contained in $\mathcal{P}_{n-1}$ as $\mathcal{P}_{n} \backslash \mathcal{P}_{n-1}$.

The amplitudes of the third iteration $t_{i j, 3}^{a b}$ will be generated in $\mathcal{P}_{2}$ according to Eqs. (11) and (21) giving the residual $R_{i j, 3}^{a b} \in \mathcal{P}_{3}$, such that more, yet untouched components, of the environment of $P$ enters the residual through the coupling mechanism described in Eq. (21). The new space components will not be coupled to $\mathcal{P}_{1}$ directly as the Fock matrix elements between these spaces are negligible, see Fig. 4. The amplitudes of the $\mathcal{P}_{3} \backslash \mathcal{P}_{2}$ space thus interact directly with the amplitudes of the $\mathcal{P}_{2} \backslash \mathcal{P}_{1}$ space which in turn couples with the amplitudes in the $\mathcal{P}_{1}$ space. Hence, the relaxation effects of the $\mathcal{P}_{3} \backslash \mathcal{P}_{2}$ space on the EOS amplitudes will be small and indirect, and these effects will therefore be referred to as secondary coupling effects.

For the fourth and following iterations, these mechanisms are preserved, i.e., additional couplings are introduced in the already considered space and new spaces are introduced which indirectly couple to the $\mathcal{P}_{1}$ space through other spaces. The effects on the EOS amplitudes from the newly introduced spaces thus become more indirect and smaller for each successive iteration.

The above developments may be summarized as follows. To evaluate the fragment energies, we need the amplitudes of the $\mathcal{P}_{\text {EOS }}^{x} \subset \mathcal{P}_{1}$ space that were introduced in iteration 1 . In the second iteration, direct coupling to the outside space $\mathcal{P}_{2} \backslash \mathcal{P}_{\text {EOS }}^{x}$ is introduced which affects the EOS amplitudes significantly. The relaxation of the EOS amplitudes through the additional components in the $\mathcal{P}_{2} \backslash \mathcal{P}_{\text {EOS }}^{x}$ space is therefore important for the evaluation of the single fragment energy. The effects of the additional spaces introduced in the following iterations affect the EOS amplitudes only indirectly. The requested precision will consequently define how much of the environment of $P$ has to be considered. Thus, we may introduce an effective coupling space $\mathcal{P}_{\mathrm{EFF}}$ where the MP2 equations are solved to give the EOS amplitudes to the requested (FOT) precision. This effective space may be defined as

$$
\mathcal{P}_{\mathrm{EFF}} \equiv[\underline{P}]_{\mathrm{EFF}}^{2} \times[\bar{P}]_{\mathrm{EFF}}^{2} .
$$

When calculating the single fragment energy, we may correspondingly replace $[\underline{P}]$ and $[\bar{P}]$ in Eqs. (3) and (5) by $[\underline{P}]_{\mathrm{EFF}}$ and $[\bar{P}]_{\mathrm{EFF}}$, giving the occupied and virtual effective EOS

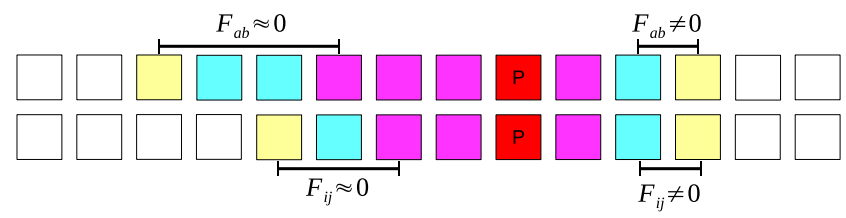

FIG. 4. Iteration 3: The residual is generated in the $\mathcal{P}_{3}$ space (all colored boxes) where the orbitals of sites with light yellow squares $\left(\mathcal{P}_{3} \backslash \mathcal{P}_{2}\right)$ symbolize the orbitals which interact directly with the orbitals of the $\mathcal{P}_{2} \backslash \mathcal{P}_{1}$ space (cyan) through non-negligible Fock matrix elements, but not directly with the orbitals of the $\mathcal{P}_{1}$ space (red and magenta).

$$
\begin{aligned}
& \mathcal{P}_{\mathrm{EOS}}^{\mathrm{o}, \mathrm{EFF}} \equiv \underline{P}^{2} \times[\bar{P}]_{\mathrm{EFF}}^{2}, \\
& \mathcal{P}_{\mathrm{EOS}}^{\mathrm{v}, \mathrm{EFF}} \equiv[\underline{P}]_{\mathrm{EFF}}^{2} \times \bar{P}^{2},
\end{aligned}
$$

which will be generically referred to as $\mathcal{P}_{\mathrm{EOS}}^{\mathrm{x}, \mathrm{EFF}}$, while $[P]_{\mathrm{EFF}}$ and $[\bar{P}]_{\mathrm{EFF}}$ will be collectively referred to as $[P]_{\mathrm{EFF}}$. The extent $[P]_{\mathrm{EFF}}$ therefore defines both $\mathcal{P}_{\mathrm{EFF}}$ (where the amplitude equations are solved) and $\mathcal{P}_{\mathrm{EOS}}^{\mathrm{x}, \mathrm{EFF}}$ (where the single fragment energy is evaluated).

\section{B. Space extension in CCSD fragment calculations}

The CCSD amplitude equations may be viewed as a nonlinear extension of the MP2 amplitude equations where the additional terms are of higher order in a Møller-Plesset perturbation analysis. ${ }^{1}$ Thus, the energetically largest contributions leading to a space extension have been considered in Section III A 2. The focus of this section is to analyze the space extensions that may be introduced in the residual of Eq. (11) by the additional terms of the CCSD amplitude equations. The CCSD singles residual in the $T_{1}$-transformed formulation may be expressed as ${ }^{1}$

$$
{ }^{\mathrm{CCSD}} R^{S}=\Omega^{A 1}+\Omega^{B 1}+\Omega^{C 1}+\Omega^{D 1},
$$

and the CCSD doubles residual reads

$$
{ }^{\mathrm{CCSD}} R^{D}=\Omega^{A 2}+\Omega^{B 2}+\Omega^{C 2}+\Omega^{D 2}+\Omega^{E 2},
$$

where the individual terms are given in Table I. The $T_{1^{-}}$ transformed integrals (denoted by a tilde in Table I) are given by $^{1}$

$$
\tilde{g}_{q r s t}=\sum_{\mu \nu \rho \sigma} \Lambda_{\mu q}^{p} \Lambda_{v r}^{h} \Lambda_{\rho s}^{p} \Lambda_{\sigma t}^{h} g_{\mu \nu \rho \sigma}
$$

TABLE I. The CCSD residual equations as given in Ref. 1. Tildes denote $T_{1}$-transformed integrals, see Eqs. (30)-(33).

Doubles terms:

$\Omega_{a i b j}^{A 2}=\tilde{g}_{a i b j}+\sum_{c d} t_{i j}^{c d} \tilde{g}_{a c b d}$

$\Omega_{a i b j}^{B 2}=\sum_{k l} t_{k l}^{a b}\left(\tilde{g}_{k i l j}+\sum_{c d} t_{i j}^{c d} \tilde{g}_{k c l d}\right)$

$\Omega_{a i b j}^{C 2}=P_{i j}^{a b}\left(1+\frac{1}{2} P_{i j}\right)\left[-\sum_{c k} t_{k i}^{b c}\left(\tilde{g}_{k j a c}-\frac{1}{2} \sum_{d l} t_{l j}^{a d} \tilde{g}_{k d l c}\right)\right]$

$\Omega_{a i b j}^{D 2}=\frac{1}{2} P_{i j}^{a b}\left[\sum_{c k} u_{j k}^{b c}\left(\tilde{L}_{a i k c}+\frac{1}{2} \sum_{d l} u_{i l}^{a d} \tilde{L}_{l d k c}\right)\right]$

$\Omega_{a i b j}^{E 2}=P_{i j}^{a b}\left[\sum_{c} t_{i j}^{a c}\left(\tilde{F}_{b c}-\sum_{d k l} u_{k l}^{b d} \tilde{g}_{l d k c}\right)\right.$

$$
\left.-\sum_{k} t_{i k}^{a b}\left(\tilde{F}_{k j}+\sum_{c d l} u_{l j}^{c d} \tilde{g}_{k d l c}\right)\right]
$$

Singles terms:

$\Omega_{a i}^{A 1}=\sum_{c d k} u_{k i}^{c d} \tilde{g}_{a d k c}$

$\Omega_{a i}^{B 1}=-\sum_{c k l} u_{k l}^{a c} \tilde{g}_{k i l c}$

$\Omega_{a i}^{C 1}=\sum_{c k} u_{i k}^{a c} \tilde{F}_{k c}$

$\Omega_{a i}^{D 1}=\tilde{F}_{a i}$

Definitions:

$P_{i j} X_{i j}^{a b}=X_{j i}^{a b}$

$P_{i j}^{a b} X_{i j}^{a b}=X_{i j}^{a b}+X_{j i}^{b a}$

$u_{i j}^{a b}=\left(2-P_{i j}\right) t_{i j}^{a b}$

$\tilde{L}_{a i b j}=\left(2-P_{i j}\right) \tilde{g}_{a i b j}$

$\tilde{F}_{p q}=\tilde{h}_{p q}+\sum_{i}\left(2 \tilde{g}_{p q i i}-\tilde{g}_{p i i q}\right)=\tilde{h}_{p q}+\sum_{\rho \sigma} \tilde{D}_{\rho \sigma}\left(2 \tilde{g}_{p q \rho \sigma}-\tilde{g}_{p \rho \sigma q}\right)$

$\tilde{D}_{\rho \sigma}=\sum_{i} \Lambda_{\rho i}^{p} \Lambda_{\sigma i}^{h}$ 
where $\mu, v, \rho$, and $\sigma$ are atomic orbital (AO) indices, and the particle $\Lambda^{p}$ and hole $\boldsymbol{\Lambda}^{h}$ transformation matrices are defined via the MO coefficient matrix $\mathbf{C}$ as

$$
\begin{aligned}
& \boldsymbol{\Lambda}^{p}=\mathbf{C}\left(\mathbf{1}-\mathbf{t}_{1}^{\mathrm{T}}\right), \\
& \boldsymbol{\Lambda}^{h}=\mathbf{C}\left(\mathbf{1}+\mathbf{t}_{1}\right),
\end{aligned}
$$

and the $\mathbf{t}_{1}$ matrix is

$$
\left[\mathbf{t}_{1}\right]_{p q}=\left\{\begin{array}{c}
t_{q}^{p} \text { if } p \text { virtual and } q \text { occupied } \\
0 \text { otherwise }
\end{array}\right\} .
$$

When the singles amplitudes are neglected, ${ }^{\mathrm{CCSD}} R^{D}$ becomes the nonlinear CCD residual ${ }^{C C D} R$ (removing the tildes in the doubles terms in Table I). The starting point for analyzing orbital space extensions for CCSD single fragment energy calculations will be an analysis of the CCD residual in Section III B 1, while we consider the effect of the CCSD singles amplitudes in Section III B 2.

For the MP2 analysis in Section III A 2, we have encountered the space extension mechanism caused by nonnegligible Fock matrix elements. For the CCD and CCSD analysis, additional space extension mechanisms can be identified. We will consider the following four mechanisms which collectively represent error type 2 of Section II:

1. Propagation due to non-negligible Fock matrix elements (identified in Section III A 2).

2. Propagation due to non-negligible charge distributions in two-electron integrals.

3. Propagation due to long-range interactions between charge distributions (dipole-dipole effects).

4. Propagation due to the Fock matrix constructed from a $T_{1}$-transformed density (charge-polarization effects).

Mechanisms 1, 2, and 3 are present for CCD and will be discussed in Section III B 1, while mechanism 4 is present only for CCSD and will be discussed in Section III B 2.

\section{Space extension mechanisms in the CCD model}

To analyse the space extension process in a CCD context, we use the framework established for MP2 in Section III A with the only modification that, when solving the nonlinear CCD equations using the conjugate residual method, the residual $R_{i j, n}^{a b}$ defined in Eq. (12) is replaced by the residual ${ }^{\mathrm{CCD}} R_{i j, n}^{a b}$.

The MP2 amplitude equations (Eq. (12)) correspond to $g_{\text {aibj }}$ in $\Omega^{A 2}$ and the Fock matrix terms of $\Omega^{E 2}$ in the CCD amplitude equations. The CCD equations thus contain additional terms where two-electron integrals are contracted linearly or quadratically with cluster amplitudes. The quadratic terms do not contribute to the space extension of the residual since all indices entering these contributions are fixed by the amplitudes of the previous iteration. For example, in the second part of the $\Omega_{n}^{B 2}$ term, $\sum_{k l} \sum_{c d} t_{k l, n}^{a b} t_{i j, n}^{c d} g_{k c l d}$, the integral indices are fixed to the space of the cluster amplitudes of iteration $n$ and therefore no space extension is introduced by this component of the residual.
As an example for the space propagation according to mechanism 2, we consider the second term of $\Omega_{a i b j, n}^{A 2}$, i.e.,

$$
\Omega_{a i b j, n}^{A 2.2}=\sum_{c d} t_{i j, n}^{c d} g_{a c b d} .
$$

At iteration $n$ the amplitudes are defined in $\mathcal{P}_{n-1}$, and the dummy indices $c$ and $d$ are therefore restricted to this space. Hence, the free indices $a, b$ of the integrals $g_{a c b d}$ are restricted by the locality of the charge distributions $\omega_{a c}$ and $\omega_{b d}$.

All terms of CCD doubles residual may be grouped into the categories following mechanism 1 or 2 except for one of the terms in $\Omega_{n}^{D 2}$, denoted as $\Omega_{n}^{D 2.1}$, which follows mechanism 3 ,

$$
\Omega_{a i b j, n}^{D 2.1}=\sum_{c k} t_{j k, n}^{b c} g_{a i k c} .
$$

Any additional space components $\mathcal{P}_{n} \backslash \mathcal{P}_{n-1}$ of iteration $n$ contribute via this term to the single fragment energy as dipole-dipole interactions with an inverse sixth power decay with the distance between center $P$ and the charge distribution $\omega_{k c}$. This may be understood from the perspective of a residual component in the EOS, $\Omega_{a i b j, n}^{D 2.1} \in \mathcal{P}_{\text {EOS }}^{x}$, which enters the single fragment energy in Eq. (14). The contribution of the new space components $\mathcal{P}_{n} \backslash \mathcal{P}_{n-1}$ where the charge distribution $\omega_{k c}$ may be located decays with its distance from $\mathcal{P}_{\text {EOS }}^{x}$ in an inverse sixth order manner because $\omega_{k c}$ occurs in Eq. (35) in both the two-electron integral and the amplitudes, both of which decay with the inverse third power ${ }^{27}$ of the distance between $\omega_{k c}$ and $P$. Furthermore, the contribution from this direct long-range modification is expected to be small since it only slightly modifies one of many residual contributions $\Omega_{a i b j, n}^{D 2.1}+\cdots \in \mathcal{P}_{\text {EOS }}^{x}$ entering Eq. (14). Based on this discussion, we expect that mechanism 3 has a small effect on the single fragment energy which becomes important only when very high precision is requested, which will be substantiated numerically in Section III C and Appendix A.

\section{Space extension mechanisms in the CCSD model with focus on the effect of singles}

In Section III B 1, we have described the progression of the space extension for the cluster amplitudes in a CCD calculation. In this section we examine the space progression for CCSD with focus on the singles amplitudes. As for the doubles amplitudes, the singles EOS amplitudes are defined by Eqs. (3) and (5). We note from Table I that the indices $i$ and $a$ of the singles amplitude equations are coupled only by mechanisms 1 and 2, and their distance decay will thus be similar to the Fock matrix and two-electron integral decays (see Appendix A). Furthermore, since the singles amplitudes enter quadratically in Eqs. (3) and (5), their direct effect on the single fragment energy is expected to be significantly smaller than the effect of the doubles amplitudes. However we need to evaluate the coupling effects introduced by the singles into the CCSD residual. For this reason, we first consider how the $T_{1}$-transformation introduces coupling effects through the two-electron integrals. After this, special attention is given to the $T_{1}$-transformed Fock matrix. 
As an illustrative example of the effect of the $T_{1^{-}}$ transformation on the two-electron integrals, we consider the integral contribution $\tilde{g}_{a i b j}$ from Table I for the occupied EOS, i.e., $\tilde{g}_{a i b j} \in \mathcal{P}_{\mathrm{EOS}}^{o}$ in an analogous argument as for the $\Omega^{D 2.1}$ term. Using Eq. (30) $\tilde{g}_{a i b j}$ may be written as

$$
\tilde{g}_{a i b j}=g_{a i b j}+\sum_{k} t_{k}^{a} g_{k i b j}+\cdots+O\left(t_{1}^{2}\right)
$$

where one example of a linear term is given and the dots indicate further linear contributions. Terms, which are quadratic and higher order in the singles amplitudes, are very small and may be neglected for the purpose of this analysis. Since we consider $\tilde{g}_{a i b j} \in \mathcal{P}_{\mathrm{EOS}}^{o}$, the linear term in Eq. (36) will only couple to the space outside $\mathcal{P}_{\text {EOS }}^{o}$ through non-negligible charge distributions $\omega_{k i}$, where the $k$ index expands through mechanism 2 and its effect on the single fragment energy quickly becomes negligible beyond $\mathcal{P}_{2}$. This analysis thus suggests that the space extensions associated with the $T_{1}$-transformed two-electron integrals are captured by the same mechanisms that describe the CCD space extension.

The $\Omega_{n}^{E 2}, \Omega_{n}^{C 1}$, and $\Omega_{n}^{D 1}$ terms have Fock matrix contributions constructed from a $T_{1}$-transformed density matrix which, using Eqs. (31) and (32), can be written as

$$
\begin{aligned}
& \tilde{F}_{\rho \sigma}=h_{\rho \sigma}+\sum_{\mu \nu} \tilde{D}_{\mu v}\left(2 g_{\rho \sigma \mu \nu}-g_{\rho v \mu \sigma}\right), \\
& \tilde{D}_{\mu v}=D_{\mu \nu}+\sum_{i a} C_{\mu i} t_{i}^{a} C_{v a},
\end{aligned}
$$

where $D_{\mu \nu}$ is the HF density matrix for the full system. The singles polarized part of the density matrix in Eq. (38) introduces long-range charge-polarization effects into the $\mathrm{AO}$ Fock matrix in Eq. (37). In a practical DEC calculation, only the singles polarization effects within the $[P]_{\mathrm{EFF}}$ space are captured. This error type was denoted mechanism 4 in the beginning of Section III B and it is only important for high precision calculations. One possible strategy for including long-range charge-polarization effects is to do as follows:

- Carry out all single fragment calculations and store the (short-ranged) singles amplitudes $t_{i}^{a}$ with $i \in \underline{P}$ and $a \in[\bar{P}]_{\mathrm{EFF}}$ for each fragment $P$.

- Collect all these contributions in a matrix $t_{1}^{\text {full }}$ with full molecular dimensions.

- Construct an approximate $T_{1}$ transformed density matrix using $t_{1}^{\text {full }}$ in Eq. (38) (full molecule).

- Construct an approximate $T_{1}$ transformed Fock matrix $\tilde{F}_{\mu \nu}$ according to Eq. (37) (full molecule).

- A second round of single (and pair) fragment calculations is carried out where the constructed Fock matrix $\tilde{F}_{\mu \nu}$ is used (using a different subset of AO indices $\{\mu, v\}$ for each fragment as described in Appendix B). In each fragment calculation $\tilde{F}_{\mu \nu}$ is kept fixed throughout the local CC iterations.

We illustrate the effect of using a long-range corrected $T_{1}$ transformed Fock matrix numerically in Section III C.

\section{Summary for the space extension in a fragment CCSD calculation}

In summary, the theoretical analysis of the present section demonstrates that, for both the MP2 and CCSD models, calculations may be carried out where the amplitude equations are solved in a subspace of the total orbital space. For the MP2 model, coupling is introduced through non-negligible Fock matrix elements (mechanism 1). For the CCD and CCSD models, which include terms of higher order in the fluctuation potential, additional coupling mechanisms have been identified. Coupling through nonnegligible charge distributions in the two-electron integrals (mechanism 2) concerns all terms in the CCSD amplitude equations except the $\Omega^{D 2.1}$ term in Eq. (35) (mechanism 3). Since the distance decay of coupling mechanism 2 is usually faster than the distance decay of the Fock matrix (see Appendix A), mechanism 2 is in general taken into account when mechanism 1 is considered. The coupling effects via long-range dipole-dipole interactions (mechanism 3 ) and the long-range polarization of the Fock matrix (mechanisms 4) are expected to contribute to the coupling error only to a small extent. We may therefore, in general, redefine the square bracket notation $[P]_{\mathrm{EFF}}$ as the orbital space that interacts with $P$ through any of the mechanisms $1-4$, and conclude that the amplitude equations may be solved in the associated subspace $\mathcal{P}_{\text {EFF }}$ of Eq. (25). The occupied $(x=o)$ or virtual $(x=v)$ fragment energies $E_{P}^{x}$ are then evaluated in $\mathcal{P}_{\mathrm{EOS}}^{\mathrm{x}, \mathrm{EFF}}$ of Eq. (26) or (27) to within the predefined precision. Furthermore, we can conclude that it may suffice to determine the fragment space $[P]_{\text {EFF }}$ for a CCSD calculation using the MP2 model, unless high precision is requested. Numerical results will be given in Section III C to substantiate this point. We also note that the perturbative triples correction to the CCSD energy, $\operatorname{CCSD}(\mathrm{T})$, may be evaluated using the same fragment spaces as for the DEC-CCSD calculation. ${ }^{31}$

Here, we have carried out the analysis of the fragment sizes for the fragments referencing a single site in Eqs. (3) and (5). We note that for the fragments referencing pairs of sites in Eqs. (4) and (6), an analogous analysis results in the same extensions due to the identical mechanisms in the MP2 and CCSD equations. The pair fragment spaces can thus be obtained as unions of single fragment spaces.

\section{Numerical support for the theoretical developments}

In this section we investigate the assumptions and conclusions from Sections III A and III B for three representative molecules with different chemical properties, (i) palmitic acid (semi-linear molecule), (ii) a glycine $\alpha$-helix (bulky molecule), and (iii) Hexadeca-1,3,5,7,9,11,13, 15-octaene (conjugated system), see Table II. The structures of these molecules are given as supplementary material. ${ }^{39}$ All calculations in this section were performed with Dunning's cc-pVDZ basis set ${ }^{36}$ using the frozen core approximation in order to have molecules with a reasonable spatial extent where it is still possible to carry out the reference calculations for the full system. The orbitals have been localized using the second power of the second moment orbitals. ${ }^{37,38}$ 
TABLE II. Test set of molecules for the single fragment optimization algorithm. See supplementary material ${ }^{39}$ for the atomic coordinates.

\begin{tabular}{llr}
\hline \hline System label & \multicolumn{1}{c}{ Chemical compound } & Basis set \\
\hline (i) & Palmitic acid & cc-pVDZ \\
(ii) & gly $_{4} \alpha$-helix & cc-pVDZ \\
(iii) & Hexadeca-1,3,5,7,9,11,13,15-octaene & cc-pVDZ \\
\hline (iv) & Lauric acid & cc-pVTZ \\
(v) & gly $_{3} \alpha$-helix & cc-pVTZ \\
(vi) & Dodeca-1,3,5,7,9,11-hexene & cc-pVTZ \\
\hline
\end{tabular}

The prerequisite for the theoretical fragment analysis of Section III was the distance decay of the two-electron integrals and the Fock matrix elements from a specific center $P$ in the molecule. This prerequisite is confirmed in Appendix A for the set of test molecules and the single fragment energies $E_{P}^{x}$ may therefore be determined to a predefined tolerance by solving the amplitude equations in a restricted orbital space $\mathcal{P}_{\text {EFF. }}$

In the following we will exemplify the sizes of coupling spaces that are necessary in actual calculations to determine the fragment energy $E_{P}^{x}$ for a set of selected atomic sites $P$. Specifically we choose as site $P$ the protonated oxygen of system (i), the $\alpha$-carbon of the $\mathrm{N}$-terminal glycine of system (ii), and one of the terminal carbons of system (iii). All sites were chosen at the rim of the molecules to better expose the distance decay behaviors of the fragment energy errors.
In Fig. 2 we have schematically illustrated how the coupling space can be identified. Below, we describe a practical realization of Fig. 2. To obtain the magenta line of Fig. 2, the absolute errors in $E_{P}^{x},\left|\Delta E_{P}^{x}\right|$ are plotted for calculations where the amplitude equations have been solved in the full orbital space $\mathcal{P}_{\text {full }}$ (all orbitals of the molecular system are included) and $[P]_{\mathrm{EFF}}$ in $\mathcal{P}_{\mathrm{EOS}}^{\mathrm{x}, \mathrm{EFF}}$ is truncated at a given distance $R_{P}$ from center $P$ (denoted Approach $\left.\mathbf{A}\right)$. For the green line in Fig. 2, a practical realization is obtained by plotting errors in $E_{P}^{x}$, where $[P]_{\mathrm{EFF}}$ is truncated at a given distance $R_{P}$ in both the space where the amplitude equations are solved $\mathcal{P}_{\mathrm{EFF}}$ and in the EOS $\mathcal{P}_{\mathrm{EOS}}^{\mathrm{x} \text {,EFF }}$ (denoted Approach $\mathbf{B}$ ). The practical errors in $E_{P}^{x}$ for both the occupied $(x=o)$ and virtual $(x=v)$ partitioning schemes are given in Fig. 5 for the three selected fragments in the upper and lower rows, respectively.

For approach A (magenta curves), the error in the single fragment energy converges rapidly and with a very similar rate with the distance $R_{P}$ for both models $\left(\mathrm{MP}^{\mathrm{A}}\right.$ or $\left.\mathrm{CCSD}^{\mathrm{A}}\right)$ and both partitioning schemes. For approach $\mathbf{B}$ (green curves), larger spaces are needed for both $\mathrm{MP}^{\mathrm{B}}$ (circles) and $\mathrm{CCSD}^{\mathrm{B}}$ (triangles) models. The $\mathrm{MP} 2^{\mathrm{B}}$ fragment energy errors are always positive and converge smoothly to the correct result, whereas the $\mathrm{CCSD}^{\mathrm{B}}$ fragment energy errors have a somewhat more erratic behavior as well as occasional artificially low errors due to changes in the sign of the $\mathrm{CCSD}^{\mathrm{B}}$ fragment energy errors when increasing the fragment orbital space. The sign of the fragment energy errors for $\mathrm{MP}^{\mathrm{B}}$ may be understood from the MP2 correlation energy expression in
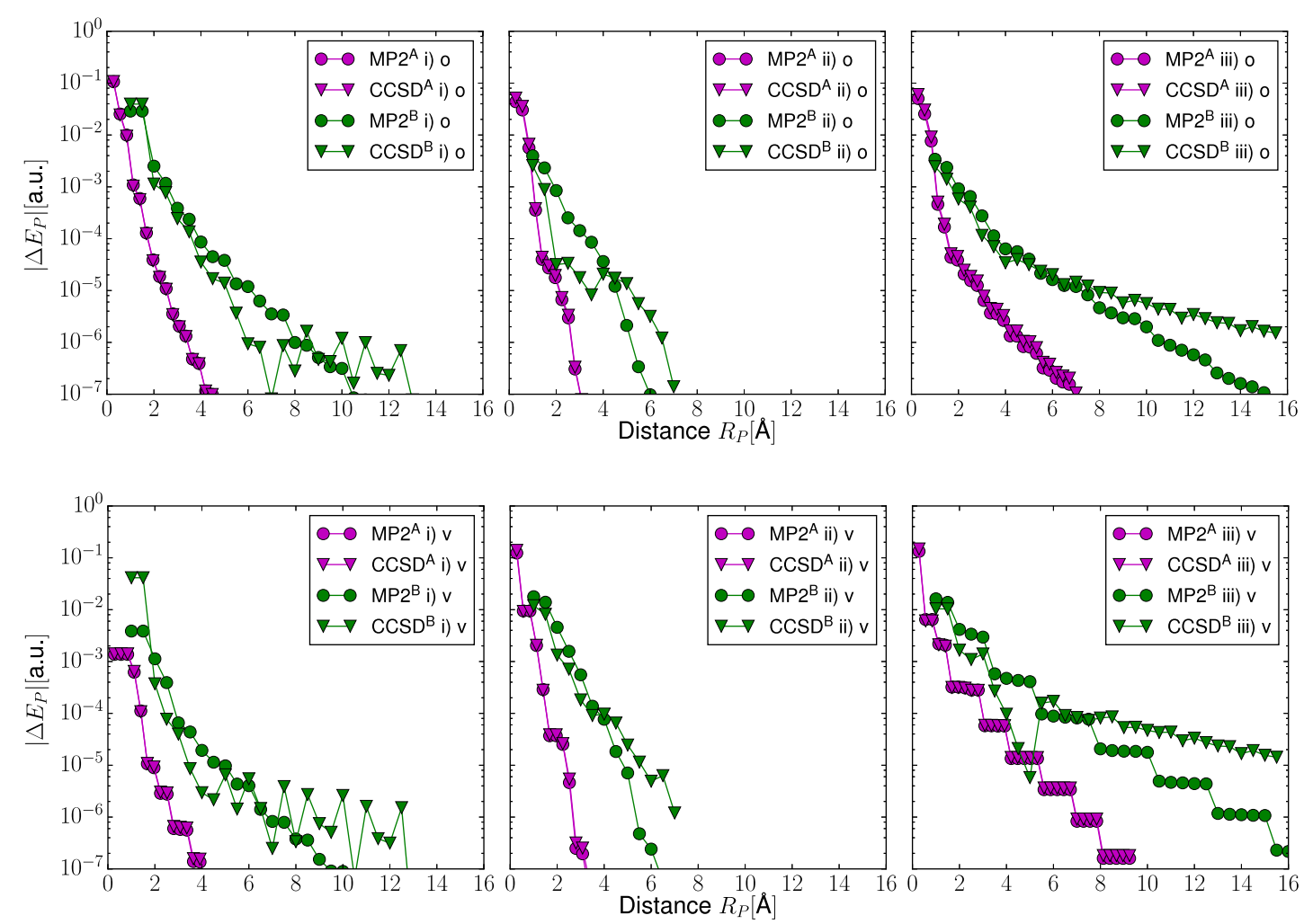

FIG. 5. Decay of the MP2 and CCSD fragment energy errors, upon increasing the orbital space for the three test systems (i), (ii), and (iii) and both the occupied (o) and virtual (v) partitioning schemes, given in the upper and lower rows, respectively. The amplitude equations have been solved in the full space (only error type 1, magenta, superscript A) and in a restricted orbital space (error type 1+2, green, superscript B). 

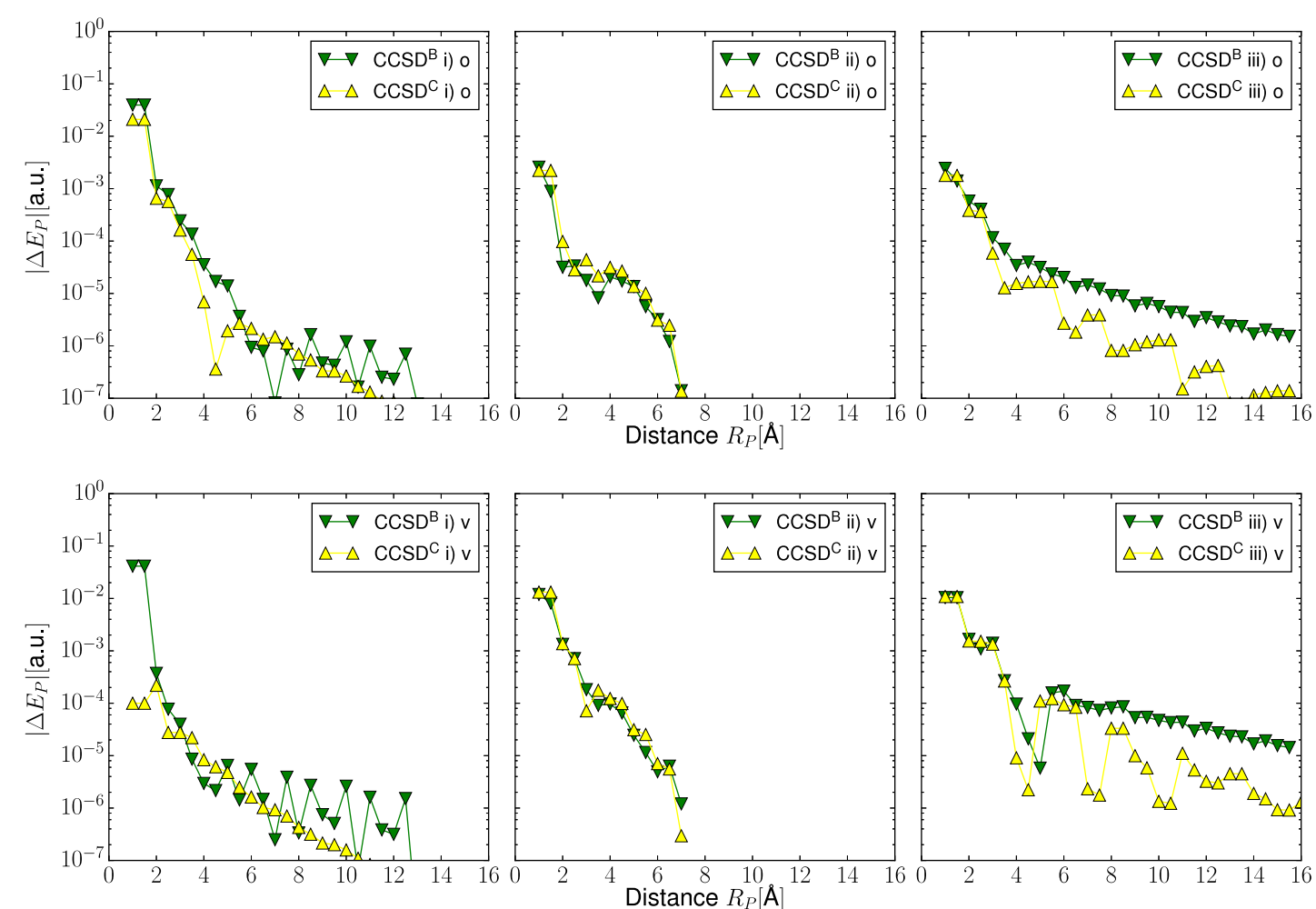

FIG. 6. Effect of the singles polarization in the Fock matrix (mechanism 4) for the three test systems (i), (ii), and (iii) for both the occupied (o) and virtual (v) partitionings, given in the upper and lower rows, respectively. The green curves $\left(\mathrm{CCSD}^{\mathrm{B}}\right)$ include the error introduced by mechanism 4 , which has been removed for the yellow curves $\left(\mathrm{CCSD}^{\mathrm{C}}\right)$ by using the exact singles polarized Fock matrix.

the canonical basis, which effectively contains a summation over squared two-electron integrals divided by negative orbital energy differences. Hence, any truncation of this expression would lead to an energy that is less negative than the true canonical MP2 energy. Although the fragment energies are evaluated in the local basis, this feature carries over to the MP2 fragment energies, i.e., any truncation leads to MP2 ${ }^{\mathrm{B}}$ fragment energies that are not negative enough. No such simple expression exists for the standard CCSD energy, which requires the solution of the non-linear CCSD amplitude equations, and the sign of the $\mathrm{CCSD}^{\mathrm{B}}$ fragment errors can therefore alternate as the fragment is expanded due to changes in the coupling environment in the CCSD fragment amplitude equations.

The horizontal difference between the green and magenta curves in Fig. 5 displays the size of the coupling space needed for a given precision. We note that the size of the coupling space depends on the model, the molecule, and the partitioning scheme. For $\left|\Delta E_{P}^{x}\right|$ values larger than $10^{-4}$ a.u., the coupling spaces are of similar size for the three test fragments. For smaller $\left|\Delta E_{P}^{x}\right|$ values, the conjugated system (iii) requires larger coupling spaces than systems (i) and (ii), in particular for the virtual partitioning scheme.

In order to study the effect of the polarization of the Fock matrix by the singles amplitudes (mechanism 4, in Section III B 2), we introduce the $\operatorname{CCSD}^{C}$ model where we eliminate errors from coupling mechanism 4 by using a singles polarized Fock matrix $\tilde{F}$ (see Table I) constructed from the converged singles amplitudes (no approximations in Eq. (38)).
In Fig. 6, we report calculations for the three test fragments for the models $\mathrm{CCSD}^{\mathrm{B}}$ and $\mathrm{CCSD}^{\mathrm{C}}$. From Fig. 6 it is evident that eliminating mechanism 4 in general decreases the CCSD fragment energy error. In particular, for the conjugated system, correcting for coupling mechanism 4 leads to reduced fragment sizes in the high-precision regime.

In summary, the calculations of the current section support the general conclusion of Sections III A and III B. Fragment energies to a given precision can be obtained by solving the $\mathrm{CC}$ amplitude equations in a restricted space when local orbitals are used, and unless high precision is requested, the fragment orbital spaces obtained at the MP2 level may be sufficient for CCSD calculations. For high-precision calculations, it was shown that it may be beneficial to account for the singles polarization of the Fock matrix in order to obtain smaller fragments. However, the investigation also shows that the coupling spaces depend on the model, the partitioning scheme, and the molecule and, in particular, that larger spaces may be necessary for the virtual partitioning scheme. In Section IV we develop a black box algorithm for determining the local fragment orbital spaces.

\section{PRACTICAL IMPLEMENTATION: DETERMINING SINGLE FRAGMENT ORBITAL SPACES}

In the theoretical locality analysis of Section III, we have identified the various coupling mechanisms that arise when MP2 and CCSD amplitude equations are solved in a 
restricted orbital space. In this section we describe a practical implementation for the determination of single fragment orbital spaces such that the single fragment energy error is of the requested (FOT) precision.

The single fragment optimization algorithm for the single fragment energy $E_{P}^{x}$ includes two main steps: the single fragment expansion procedure where $[P]_{\mathrm{EFF}}$ is increased until the energy difference between the last two expansion steps is lower than the FOT, and the reduction procedure where $[P]_{\mathrm{EFF}}$ is fine tuned (reduced) without compromising the precision of the single fragment energy.

For the discussion of the single fragment optimization procedure, it is convenient to introduce the reference single fragment energies $E_{P}^{x, f}$, evaluated from $\mathcal{P}_{\text {EOS }}^{\text {x,full }}$ (all orbitals included in Eqs. (26) and (27))

$$
\begin{aligned}
& E_{P}^{o, f}=\sum_{i, j \in \underline{P}} \sum_{a b} \tau_{i j}^{a b, f} L_{i a j b}, \\
& E_{P}^{v, f}=\sum_{i j} \sum_{a, b \in \bar{P}} \tau_{i j}^{a b, f} L_{i a j b},
\end{aligned}
$$

where the amplitudes $\tau_{i j}^{a b, f}$ are obtained from $\mathcal{P}_{\text {full }}$.

\section{A. Expansion of the orbital space}

In the first step of the single fragment expansion algorithm, the local orbitals are ordered according to a measure that estimates their contribution to the single fragment energy $E_{P}^{x}$. Based on the analysis in Section III, a simple yet reasonable measure is the distance between the orbital's center of charge and the center $P$ of the single fragment. In each expansion step a predefined number of orbitals (vide infra) is added to the single fragment orbital space $[P]_{\mathrm{EFF}}$. The single fragment expansion is illustrated in Fig. 7 (left). In each expansion step, the energy is evaluated at the requested level of theory using Eqs. (3) and (5), and the energy difference between two subsequent steps $i$ and $i+1$ is calculated,

$$
\delta_{e} E_{P}^{x}(i)=\left|E_{P}^{x}(i)-E_{P}^{x}(i+1)\right| .
$$

The expansion procedure continues until $\delta_{e} E_{P}^{x}(n-1)<$ FOT, and the energy contribution from orbitals not included in $[P]_{\mathrm{EFF}}$ of step $n$ is therefore assumed to be below the requested precision. In the following, we consider the space of expansion step $n,[P]_{\mathrm{EFF}}^{n}$, as the reference space $[P]_{\mathrm{REF}}$ and its associated energy, $E_{P}^{x}(n)$, as the reference energy $E_{P}^{\mathrm{REF}}$ for the single fragment reduction procedure. Thus, for the requested (FOT) precision, we assume $\left|E_{P}^{\mathrm{REF}}-E_{P}^{x, f}\right| \ll \mathrm{FOT}$, which is a reasonable assumption due to the rapid decay of the single fragment energy with respect to the inclusion of additional orbitals provided enough orbitals are included in each step, see, e.g., Fig. 5. In order for $\left|E_{P}^{\mathrm{REF}}-E_{P}^{x, f}\right| \ll$ FOT to hold, the number of orbitals added in each expansion step needs to be large enough to avoid false convergence, but also small enough to avoid that the expanded orbital space $[P]_{\text {REF }}$ becomes unacceptably large. In practice we include $5 \cdot n_{\text {orb }} / n_{\text {atoms }}$ orbitals in each expansion step, where $n_{\text {atoms }}$ is the number of atoms and $n_{\text {orb }}$ is the number of orbitals in the molecule. Extensive testing has shown that this choice is a reasonable compromise between accuracy and computational cost.

\section{B. Reduction of the orbital space}

The reduction step defines the final single fragment orbital spaces and thus the pair fragment orbital spaces which are obtained as unions of single fragment spaces. Since the pair fragment calculations dominate the total DEC calculation, it is crucial to reduce the single fragment sizes as much as possible within the chosen FOT precision to reduce the cost of the total calculation. To achieve this, a binary search algorithm is used in connection with a priority list of orbitals where the energy of the expanded single fragment, $E_{P}^{\mathrm{REF}}$, serves as a reference. For the calculations presented in Section V, we have chosen the same distance ordered list as in the expansion procedure. This is an obvious choice but other lists that produce similar results have also been tested (e.g., a list based on orbital contributions to the single fragment energy, or the absolute size of Fock matrix elements). The priority list is used to remove the least important orbitals of the $[P]_{\text {REF }}$ orbital space in the reduction procedure, see Fig. 7 (right). After each step $j$ in the binary search algorithm, the energy is evaluated and compared to the energy of the reference single fragment,

$$
\delta_{r} E_{P}^{x}(n+j)=\left|E_{P}^{x}(n+j)-E_{P}^{\mathrm{REF}}\right| .
$$

The step is accepted if

$$
\delta_{r} E_{P}^{x}(n+j)<\mathrm{FOT},
$$

Fragment expansion

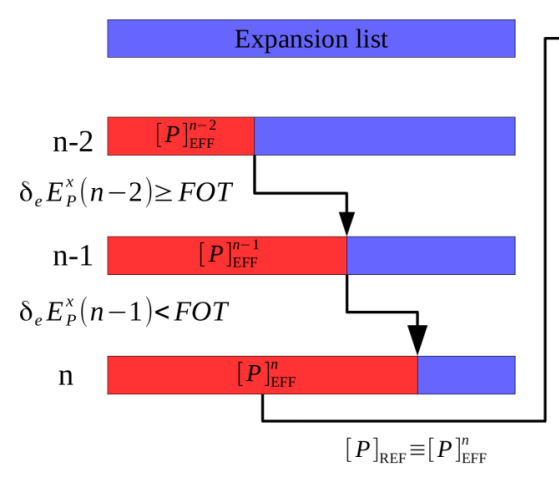

Fragment reduction - Binary search

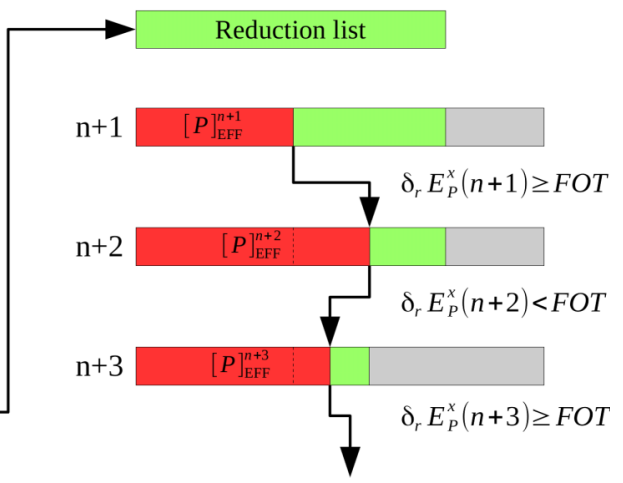

FIG. 7. The general single fragment optimization algorithm consists of two steps. In the single fragment expansion procedure (left), a reference orbital space is determined based on Eq. (41). In the reduction procedure (right), this reference orbital space is fine-tuned using a binary search algorithm where the step direction is based on Eq. (42). Red: Orbitals included in the current single fragment. Blue: Expansion priority list of orbitals. Green: Reduction priority list of orbitals. Grey: Discarded orbitals. 
and in this case the next step is chosen to further decrease the single fragment size. On the other hand, if $\delta E_{P}^{x} \geq$ FOT, the next step is chosen to increase the single fragment size, see Fig. 7. This process is repeated until the orbital space $[P]_{\mathrm{EFF}}$ does not change significantly, e.g., if the difference in the number of orbitals between two subsequent steps is less than $5 \%$ of the number of orbitals in $[P]_{\text {REF. The final single }}$ fragment orbital space is the one that corresponds to the last accepted step of the reduction procedure.

\section{Summary and cost reduction considerations}

The single fragment optimization algorithm in Fig. 7 is by no means unique but it is a model- and molecule-independent and easily programmable black-box approach, which ensures that each single fragment energy has a precision corresponding to the input FOT and thus ultimately defines the precision of the total calculation. We also note, that the single fragment optimization may be performed for $x=o, x=v$, or both simultaneously.

In order to reduce the cost of the single fragment optimization, it can be performed at a lower level of theory than that of the target model. For example, for a DEC-CCSD calculation, it may be possible to determine the single fragment orbital spaces by applying the procedure in Fig. 7 to the MP2 model. As will be described in Section V, this is a feasible approach for a typical FOT (e.g., FOT $=10^{-4}$ a.u.), while for high-precision work, a reliable DEC-CCSD energy requires that the CCSD model is also used for the single fragment optimization algorithm in Fig. 7, in particular if the virtual partitioning scheme is used. In this case, the convergence of the single fragment optimization procedure is evaluated based on both the CCSD and the MP2 single fragment energies (MP2/CCSD) in order to avoid false convergence that might
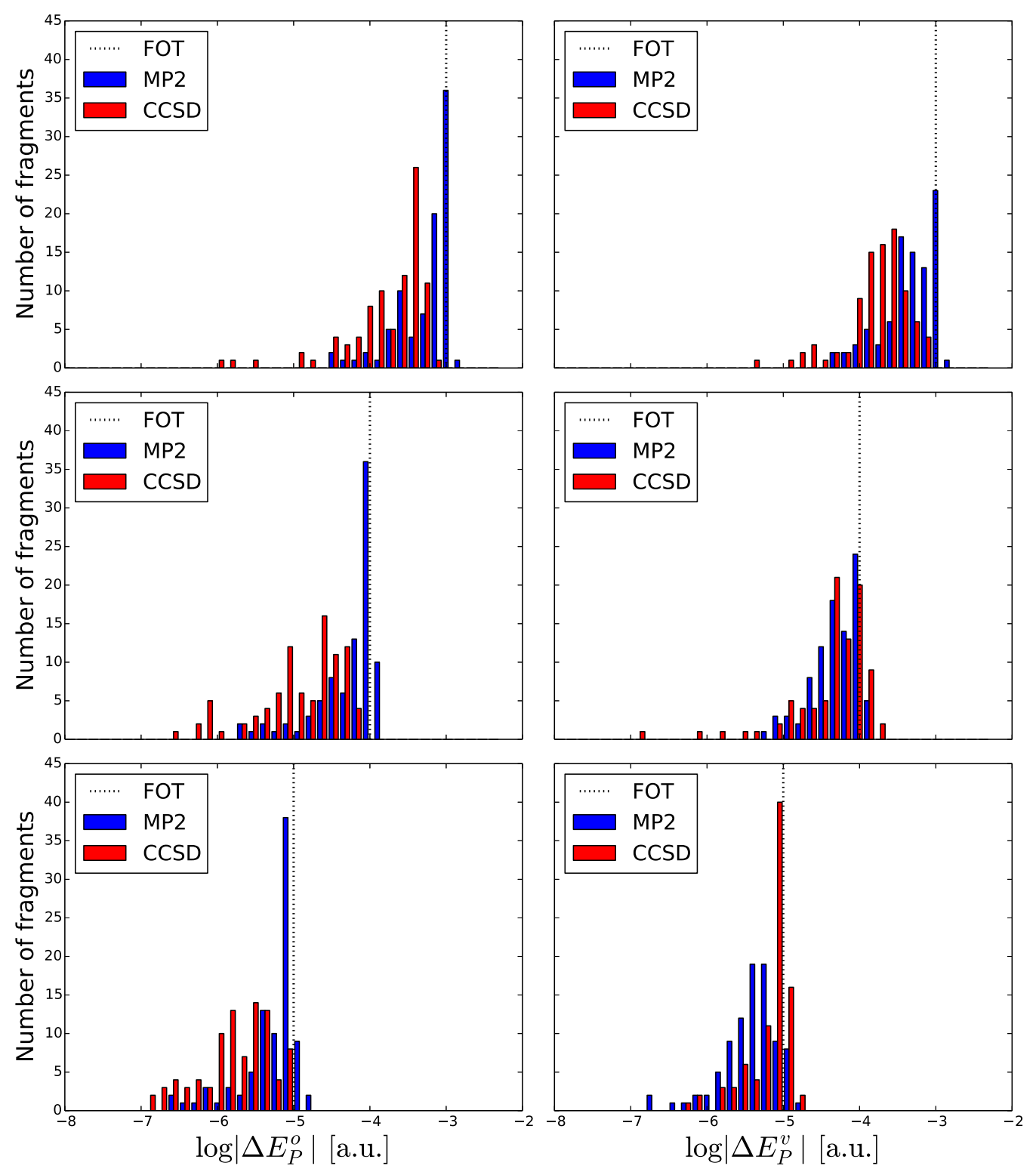

FIG. 8. Distribution of the absolute single fragment energy errors $\left|\Delta E_{P}^{x}\right|$ with respect to calculations in the full orbital spaces for both occupied ( $x=o$, left column) and virtual ( $x=v$, right column) partitioning schemes. The DEC single fragment orbital spaces have been optimized at the MP2 and MP2/CCSD levels of theory to obtain the MP2 and CCSD single fragment energy errors, respectively. Top panel: FOT $=10^{-3}$ a.u.; middle panel: FOT $=10^{-4}$ a.u.; bottom panel: FOT $=10^{-5}$ a.u. 
occur because the sign of the CCSD errors might alternate during the expansion/reduction procedure (MP2 errors are in general positive). It is also possible to further reduce the cost by invoking, e.g., the resolution-of-the-identity (RI) in the single fragment optimization algorithm. ${ }^{32}$

Finally, we note that in each fragment calculation, the AO space is truncated by fitting the MOs as described in Appendix B. This reduces the number of AO integrals that need to be evaluated.

\section{NUMERICAL ILLUSTRATIONS}

The algorithm devised in Fig. 7 is a black-box method that operates based on the locality considerations of Section III. In this section we numerically demonstrate that this algorithm leads to single fragment energies with the requested precision.
We have performed a series of calculations on six different systems using Dunning's correlation consistent cc-pVDZ and cc-pVTZ basis sets ${ }^{36}$ (see Table II and supplementary material $^{39}$ for the atomic coordinates), and for the orbital localization, we have used the second power of the second moment localized orbitals. ${ }^{37,38}$ For all calculations, the single fragment optimization in Fig. 7 was carried out for both partitioning schemes simultaneously. The calculations used the frozen core approximation and were carried out using a local version of the LSDALTON program. ${ }^{40,41}$ For the CCSD calculations, we have not used the long-range corrected Fock matrix.

In Fig. 8, we have plotted the distributions of the numerical values of the single fragment energy errors $\left|\Delta E_{P}^{x}\right|=\left|E_{P}^{x}-E_{P}^{x, f}\right|$, where $E_{P}^{x, f}$ is given by Eqs. (39) and (40) and requires calculations on the full molecular systems. This investigation is therefore limited to molecules for which the
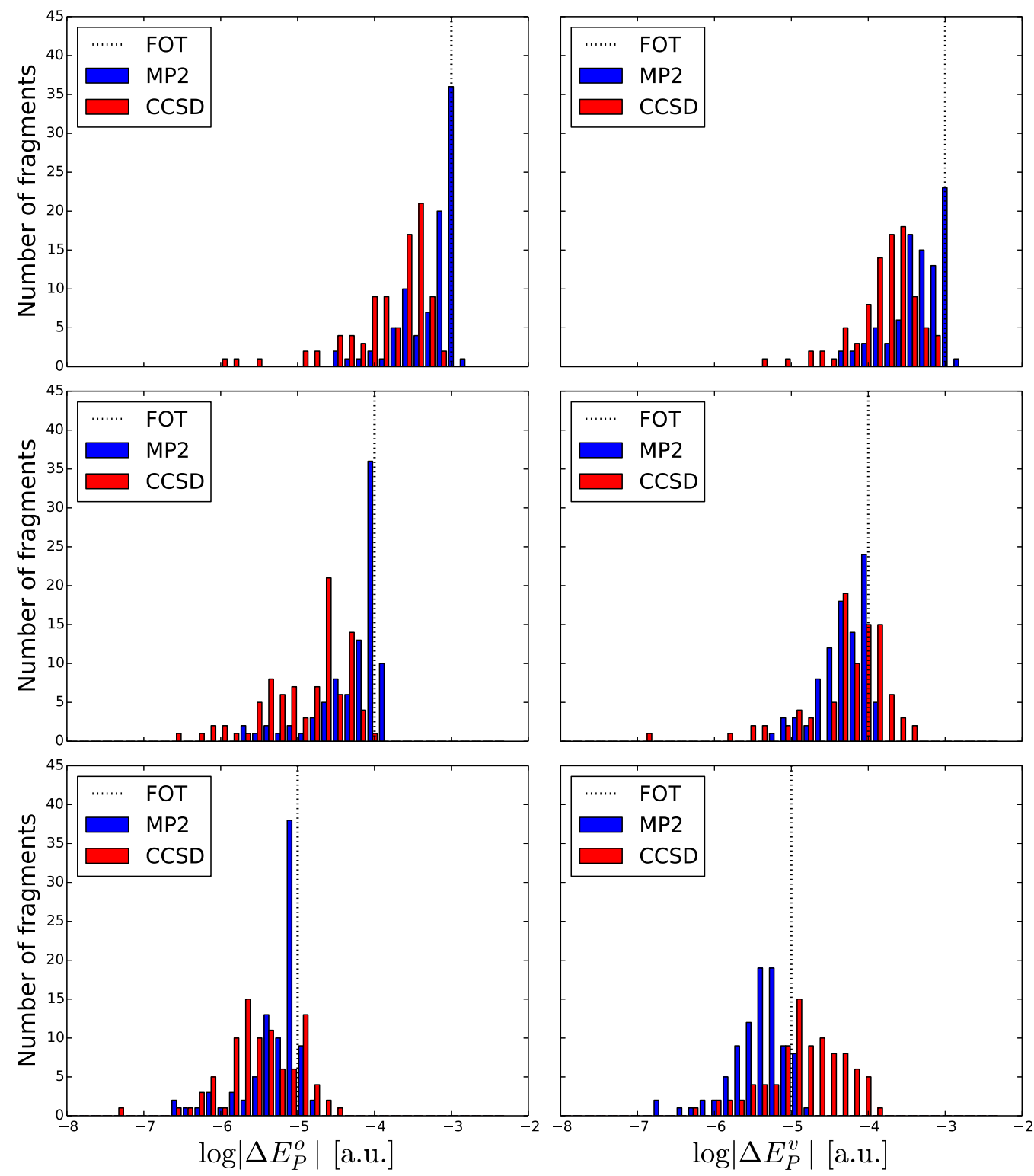

FIG. 9. Distribution of the absolute single fragment energy errors $\left|\Delta E_{P}^{x}\right|$ with respect to calculations in the full orbital spaces for both occupied ( $x=o$, left column) and virtual ( $x=v$, right column) partitioning schemes. The DEC single fragment orbital spaces have been optimized at the MP2 level of theory to obtain the MP2 and CCSD single fragment energy errors. Top panel: FOT $=10^{-3}$ a.u.; middle panel: FOT $=10^{-4}$ a.u.; bottom panel: FOT $=10^{-5}$ a.u. 
full calculation is feasible at the MP2 and CCSD levels of theory. The orbital spaces $\left([P]_{\mathrm{EFF}}\right)$ for the calculations presented in Fig. 8 were optimized at the MP2 level of theory for the MP2 single fragment energy errors, and at the combined MP2/CCSD level of theory for the CCSD single fragment energy errors using the algorithm in Fig. 7 and for decreasing values of the FOT (top panels $10^{-3}$ a.u., middle panels $10^{-4}$ a.u., lower panels $10^{-5}$ a.u.). In general, the single fragment optimization procedure leads to errors that are of the requested precision, but errors slightly larger than the FOT may occur, because the single fragment reduction procedure in Fig. 7 relies on the assumption $\left|E_{P}^{\mathrm{REF}}-E_{P}^{x, f}\right| \ll$ FOT, see Section IV A. However, in general Fig. 8 shows that when the FOT is specified, the expansion-reduction procedure in Fig. 7 gives single fragment energies that are of the size of the FOT (or smaller).

In Fig. 9, we have plotted the distributions of the numerical values of the single fragment energy errors $\left|\Delta E_{P}^{x}\right|=\left|E_{P}^{x}-E_{P}^{x, f}\right|$ when the orbital spaces $[P]_{\mathrm{EFF}}$ have been optimized at the MP2 level of theory to obtain both the MP2 and CCSD single fragment energy errors using the algorithm in Fig. 7 and for decreasing values of the FOT. The MP2 single fragment energy errors are of course the same as in Fig. 8. However, the CCSD single fragment energy errors are not as well-behaved as in Fig. 8. For example, for the virtual partitioning scheme, the CCSD errors are in general below the requested precision for $\mathrm{FOT}=10^{-3}$ a.u., while single fragment energy errors of $5 \cdot 10^{-4}\left(1 \cdot 10^{-4}\right)$ a.u. occur for a FOT of $10^{-4}\left(10^{-5}\right)$ a.u. For the occupied partitioning scheme, the single fragment energy errors are surprisingly well-behaved and the CCSD single fragment energy errors are in general of the size of the FOT. From a pragmatic point of view, an important implication is that for the FOTs of practical interest considered here, the local orbital spaces determined at the MP2 level of theory can also be used for CCSD calculations, if only the occupied partitioning is used. This leads to significant savings compared to carrying out the single fragment optimization at the CCSD level of theory, particularly for the largest single fragment calculation (the $n$th step in Fig. 7). It is, however, important to emphasize that a DEC-CCSD calculation with rigorous error control requires that the single fragment optimization is carried out at the combined MP2/CCSD level of theory. This becomes especially important when both partitioning schemes are needed in order to calculate molecular gradients. ${ }^{28}$

As a last point of this section, we would like to emphasize the importance of the second part of the single fragment optimization algorithm in Fig. 7, the single fragment reduction procedure. A comparison of the size of the orbital space before and after the single fragment reduction shows a reduction of the occupied space by $28 \%$ on average, while the virtual space is typically reduced by $35 \%$ to $40 \%$. In terms of a $V^{4} O^{2}$ scaling CCSD algorithm, that corresponds to a mean cost reduction of each fragment calculation by more than one order of magnitude. Such savings are crucial to reduce the computational cost for the pair fragments (unions of single fragment spaces) which dominate the calculation.

In conclusion, the numerical data provided in this section support the theoretical analysis in Section III and show that the algorithm in Fig. 7 is a useful practical implementation for determining local orbital spaces.

\section{CONCLUSION}

In this article we have carried out a theoretical locality analysis and a subsequent practical implementation to demonstrate how the nonlinear set of CCSD amplitude equations for DEC fragment calculations may be solved to within a desired precision using a restricted set of local HF orbitals. A practical algorithm for determining the local orbital spaces needed to obtain the single fragment (and hence pair fragment) energies to within the requested precision has been implemented based on the principles identified in the theoretical analysis. The practical algorithm is focused on determining the smallest possible single fragment spaces (within the desired precision) in order to reduce the cost for the time-dominating pair fragment calculations as much as possible. We have finally demonstrated the numerical effectiveness and robustness of the single fragment optimization algorithm. In short, a solid theoretical foundation for local CCSD calculations and the identification of local orbital spaces has been established.

With the theoretical analysis, we have identified two error sources that are present when the single fragment energy is evaluated in a restricted orbital space, i.e., (a) the direct energy contribution of omitted orbitals and (b) the relaxation effects of omitted space components when solving the amplitude equations. When solving the CCSD amplitude equations in a restricted orbital space, four distinct relaxation mechanisms have been identified, i.e., a relaxation through (1) nonvanishing Fock matrix elements, (2) non-vanishing charge distributions in the two-electron integrals, (3) long-range (dipole-dipole) contributions in the two-electron integrals, and (4) long-range (charge-polarization) contributions from the $T_{1}$-transformed Fock matrix. Mechanism (1) was found to be the dominant mechanism, and only when higher precision is requested (FOT $<10^{-4}$ a.u.) mechanisms (2)-(4) become important. It was demonstrated that the effects of mechanisms (2) and (3) had a similar spatial extent as mechanism (1), while the effects of mechanism (4) could be partially remedied by constructing an approximate long-range $T_{1}$ transformed density matrix with singles amplitude contributions from all single fragments. For the occupied partitioning scheme, the single fragment orbital spaces may be determined at the MP2 level for a DEC-CCSD calculation, while, for the virtual partitioning scheme, it is in general necessary to determine the single fragment orbital spaces at the CCSD level to ensure robustness of the results. In general, the theoretical and numerical results thus demonstrate that the local orbital spaces are model- and molecule-dependent, underlining the importance of using a black-box algorithm for determining the local orbital spaces.

\section{ACKNOWLEDGMENTS}

The research leading to these results has received funding from the European Research Council under the 
European Union's Seventh Framework Programme (FP/20072013)/ERC Grant Agreement No. 291371.

This research used resources of the Oak Ridge Leadership Computing Facility at Oak Ridge National Laboratory, which is supported by the Office of Science of the Department of Energy under Contract No. DE-AC05-00OR22725.

We also acknowledge the PRACE Research Infrastructure resource Curie at the Très Grand Centre de Calcul (TGCC) operated by CEA near Paris, France.

\section{APPENDIX A: CHECKING THE INITIAL CONDITIONS OF THE FRAGMENT ANALYSIS}

The prerequisites for the theoretical fragment analysis in Section III were that the two-electron integrals and the Fock matrix decay with the distance seen from a specific center $P$ in the molecule. In this appendix, we numerically verify this assumption. In Fig. 10, we display the decay of the Fock matrix and the two-electron integrals from the perspective of the centers used in Section III C. For simplifying the plots, only the maximum value within intervals of $1.3 \AA$ is given.

From Fig. 10, it is evident that the initial assumptions of Section III hold for all three chosen fragments, although there are minimal differences for the systems. Note that for all three systems, the virtual-virtual block (blue circles) of the Fock matrix (mechanism 1) has the largest contributions at a given distance. The observed difference between the charge distribution decay (red pluses, mechanism 2) and the long-range dipole-dipole decay (cyan triangles, mechanism 3 ) of the two-electron integrals for the three fragments under investigation is minimal.

In Fig. 11 we compare the (charge distribution) decay of the singles (blue circles) and doubles (green $\mathrm{x}$ ) amplitudes for the three fragments under investigation. As expected, the doubles amplitude decays roughly follow the decay behaviours of the corresponding two-electron integrals (mechanism 2) in Fig. 10 and thus the decay is slowest for system (iii).

\section{APPENDIX B: DEFINING LOCALITY OF MOLECULAR ORBITALS AND SINGLE FRAGMENT EXTENTS}

In a DEC calculation, a localized HF MO $\phi_{r}^{P}$ is assigned to the atomic site $P$ where its Löwdin atomic charge $Q_{\text {atom }}^{r}$ is largest. In case two atoms have similar Löwdin atomic charges, it is not important which atom a local orbital is assigned to, since the fragment optimization procedure ensures that the local orbital spaces are adapted to the specific orbital assignment, such that the atomic fragment energy is determined to the requested precision. Even though the bulk of $\phi_{r}^{P}$ is confined to a small volume of space, $\phi_{r}^{P}$ has small
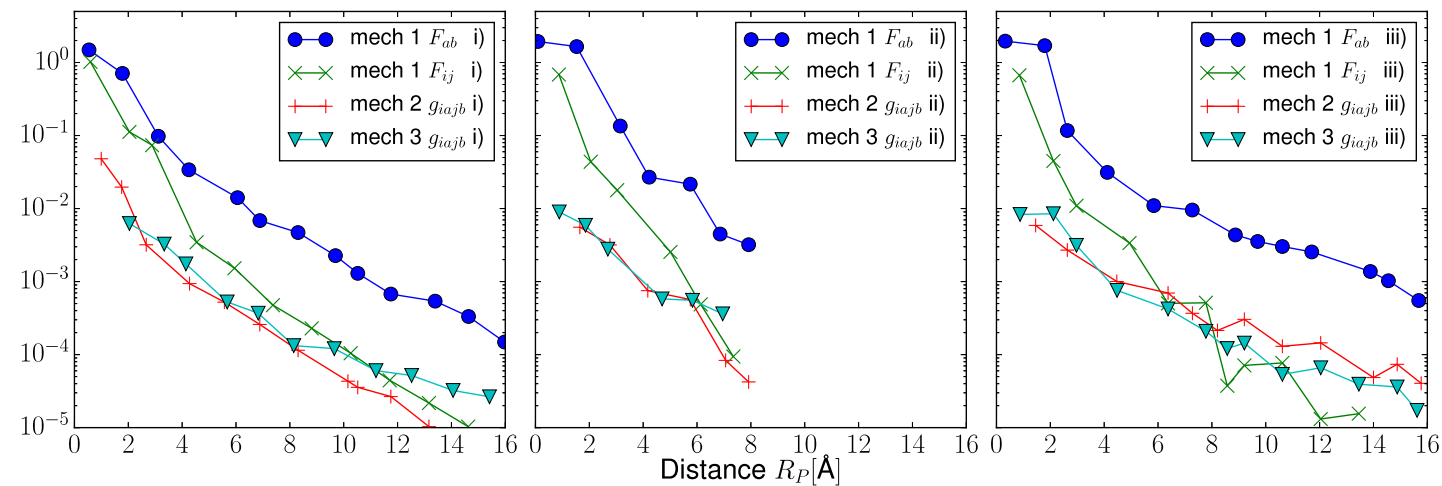

FIG. 10. The decay of the occupied-occupied Fock matrix elements $F_{i j}$ with $i \in P, j \in[P]$ (mechanism 1, green x) and the virtual-virtual Fock matrix elements $F_{a b}$ with $a \in P, b \in[P]$ (mechanism 1, blue circles) with the distance from the chosen sites $P$ for systems (i), (ii), and (iii) is compared to the charge distribution decay in the two-electron integrals $g_{a i b j}$ with $i, j, b \in P, a \in[P]$ (mechanism 2, red pluses) and the long-range dipole-dipole decay in $g_{a i b j}$ with $i, a \in P, j, a \in[P]$ (mechanism 4, cyan triangles).
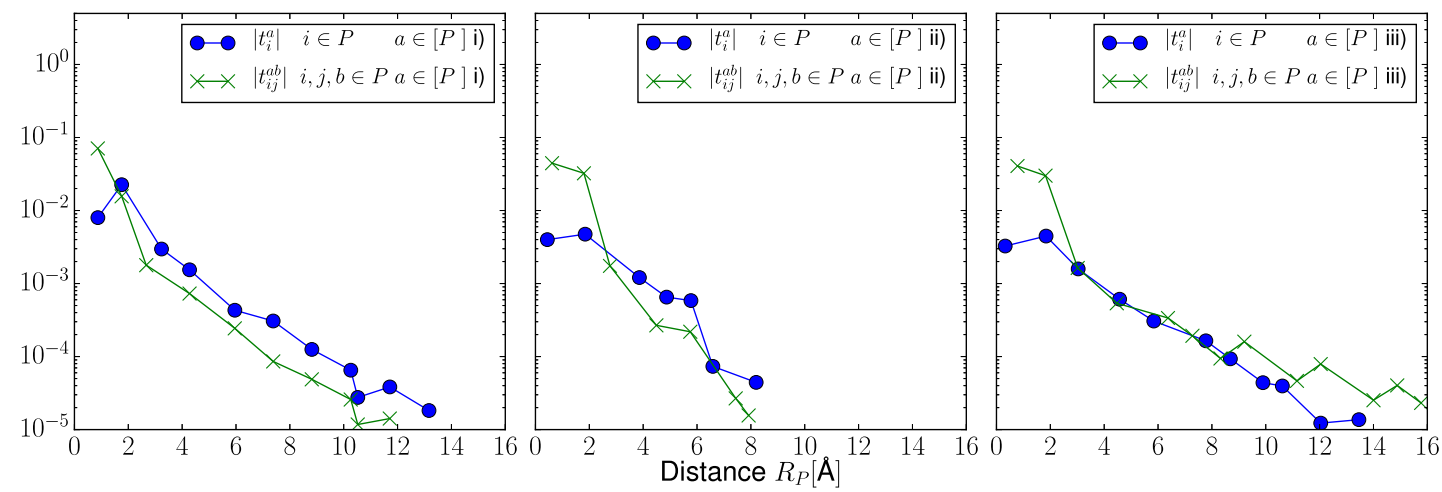

FIG. 11. The decay behaviours of the singles (blue circles) and doubles (green x) amplitudes with the distance to the chosen sites $P$ of systems (i), (ii), and (iii). 
expansion coefficients on AOs located some distance away from $P$. In this appendix we describe how this tail region may be treated without considering explicitly the AOs at which the small expansion coefficients are situated. This development is used to reduce the number of AOs and thereby the number of AO integrals which have to be evaluated. The procedure described here is very similar to the one described in Section 6.1 of Ref. 26. However, there are subtle differences, and for completeness the current implementation of the procedure is summarized here.

The HF orbital $\phi_{r}^{P}$,

$$
\phi_{r}^{P}=\sum_{\mu} \chi_{\mu} c_{\mu r}^{P},
$$

may be approximated in the following way:

$$
\tilde{\phi}_{r}^{P}=\sum_{\tilde{\mu}} \chi_{\tilde{\mu}} \tilde{c}_{\tilde{\mu} r}^{P},
$$

where the $\tilde{\mu}$-summation is restricted to AOs which in some sense are neighboring the atomic site $P$ (to be detailed below). The expansion coefficients of $\tilde{\phi}_{r}^{P}$ may be determined from a least squares fit, i.e., by minimizing the function $f\left(\tilde{\mathbf{c}}^{P}\right)$,

$$
f\left(\tilde{\mathbf{c}}^{P}\right)=\left\|\tilde{\phi}_{r}^{P}-\phi_{r}^{P}\right\|=\left\langle\tilde{\phi}_{r}^{P}-\phi_{r}^{P} \mid \tilde{\phi}_{r}^{P}-\phi_{r}^{P}\right\rangle,
$$

with respect to the $\tilde{c}$ coefficients. This gives the expansion coefficients

$$
\tilde{c}_{\tilde{\mu} r}^{P}=\sum_{\tilde{\nu} \eta}(\tilde{\mathbf{S}})_{\tilde{\mu} \tilde{v}}^{-1} S_{\tilde{\nu} \eta} c_{\eta r}^{P}
$$

where the dimensions of the overlap matrices are defined by the restrictions that are imposed on the $\mathrm{AO}$ indices, i.e.,

$$
\begin{aligned}
\tilde{S}_{\tilde{\mu} \tilde{v}} & =\left\langle\chi_{\tilde{\mu}} \mid \chi_{\tilde{v}}\right\rangle, \\
S_{\tilde{v} \eta} & =\left\langle\chi_{\tilde{v}} \mid \chi_{\eta}\right\rangle .
\end{aligned}
$$

For each MO $\phi_{r}^{P}$, a prioritized list of AOs may be generated by quantifying the importance of each $\mathrm{AO} \chi_{\mu}$ according to its Löwdin charge $Q_{\mu}^{r}$, noting that the sum over all Löwdin charges for MO $\phi_{r}^{P}$ equals one. We include AOs from this list until one minus the sum of Löwdin charges is smaller than a given threshold $\delta$,

$$
1-\sum_{\tilde{\mu}} Q_{\tilde{\mu}}^{r}<\delta
$$

where $\delta$ is a small prefixed number. This procedure defines a set of AOs for each MO $\phi_{r}^{P}$, which we denote the orbital extent $\left\{\phi_{r}^{P}\right\}$. The union of orbital extents for all MOs in the effective orbital space $[P]_{\mathrm{EFF}}$ is denoted the atomic fragment extent $\{P\}$. The $\{P\}$ space defines the set of AOs used to describe the MOs in single fragment $P$, i.e., it defines the restriction on the $\tilde{\mu}$-summation in Eq. (B2). Thus, all MOs in the fragment are fitted using the same set of AOs to ensure a uniform description. We note that a screening of atomic centers in accordance with Eq. (B7) was used by Boughton and Pulay ${ }^{42}$ for the occupied HF orbitals as a completeness criteria for the assignment of local excitation spaces.

In practice we use $\delta=0.05$ to define the orbital extents. This might appear to be a very crude value; however, the effect of approximating the MOs is minor because they are fitted using the union of all orbital extents. In particular, for the MOs close to site $P$ (large single fragment energy contributions), the fitting procedure has virtually no effect, while it slightly modifies the MOs far from $P$ (small single fragment energy contributions). The fitting procedure therefore has a very minor effect on the single fragment energy. Furthermore, each step of the fragment expansion in Fig. 7 not only includes new energy contributions and new coupling effects by including more MOs, but it also improves the description of the MOs already included in the previous fragment $(\{P\}$ is enlarged). The effect of the approximation in Eq. (B2) is thus automatically taken into account by the fragment optimization procedure.

${ }^{1}$ T. Helgaker, P. Jørgensen, and J. Olsen, Molecular Electronic Structure Theory, 1st ed. (Wiley, Chichester, England, 2000).

${ }^{2}$ P. Pulay, Chem. Phys. Lett. 100, 151 (1983).

${ }^{3}$ S. Saeb $\varnothing$ and P. Pulay, Annu. Rev. Phys. Chem. 44, 213 (1993).

${ }^{4}$ C. Hampel and H.-J. Werner, J. Chem. Phys. 104, 6286 (1996).

${ }^{5}$ M. Schütz, G. Hetzer, and H.-J. Werner, J. Chem. Phys. 111, 5691 (1999).

${ }^{6}$ P. Y. Ayala and G. E. Scuseria, J. Chem. Phys. 110, 3660 (1999).

${ }^{7}$ V. Weijo, P. Manninen, P. Jørgensen, O. Christiansen, and J. Olsen, J. Chem. Phys. 127, 074106 (2007).

${ }^{8}$ N. Flocke and R. J. Bartlett, J. Chem. Phys. 121, 10935 (2004)

${ }^{9} \mathrm{~S}$. Li, J. Ma, and Y. Jiang, J. Comput. Chem. 23, 237 (2002).

${ }^{10}$ Z. Rolik and M. Kállay, J. Chem. Phys. 135, 104111 (2011).

${ }^{11}$ W. Li, Y. Guo, and S. Li, Phys. Chem. Chem. Phys. 14, 7854 (2012).

${ }^{12} \mathrm{~W}$. Li and S. Li, J. Chem. Phys. 121, 6649 (2004).

${ }^{13}$ M. Kobayashi and H. Nakai, J. Chem. Phys. 129, 044103 (2008).

${ }^{14}$ D. G. Fedorov and K. Kitaura, J. Chem. Phys. 123, 134103 (2005).

${ }^{15}$ D. G. Fedorov, T. Nagata, and K. Kitaura, Phys. Chem. Chem. Phys. 14, 7562 (2012).

${ }^{16}$ H. Stoll, Chem. Phys. Lett. 191, 548 (1992).

${ }^{17}$ J. Friedrich, M. Hanrath, and M. Dolg, J. Chem. Phys. 126, 154110 (2007).

${ }^{18}$ M. Häser and J. Almlöf, J. Chem. Phys. 96, 489 (1992).

${ }^{19}$ B. Doser, D. S. Lambrecht, and C. Ochsenfeld, Phys. Chem. Chem. Phys. 10, 3335 (2008).

${ }^{20}$ S. Schweizer, B. Doser, and C. Ochsenfeld, J. Chem. Phys. 128, 154101 (2008).

${ }^{21}$ F. Neese, F. Wennmohs, and A. Hansen, J. Chem. Phys. 130, 114108 (2009).

${ }^{22}$ F. Neese, A. Hansen, and D. G. Liakos, J. Chem. Phys. 131, 064103 (2009).

${ }^{23}$ C. Riplinger and F. Neese, J. Chem. Phys. 138, 034106 (2013).

${ }^{24}$ J. Yang, G. K. L. Chan, F. R. Manby, M. Schütz, and H.-J. Werner, J. Chem. Phys. 136, 114105 (2012)

${ }^{25}$ M. Ziółkowski, B. Jansík, T. Kjærgaard, and P. Jørgensen, J. Chem. Phys. 133, 014107 (2010).

${ }^{26} \mathrm{~K}$. Kristensen, M. Ziółkowski, B. Jansík, T. Kjærgaard, and P. Jørgensen, J. Chem. Theory Comput. 7, 1677 (2011).

${ }^{27}$ I.-M. Høyvik, K. Kristensen, B. Jansík, and P. Jørgensen, J. Chem. Phys. 136, 014105 (2012)

${ }^{28}$ K. Kristensen, P. Jørgensen, B. Jansík, T. Kjærgaard, and S. Reine, J. Chem. Phys. 137, 114102 (2012).

${ }^{29}$ K. Kristensen, I.-M. Høyvik, B. Jansík, P. Jørgensen, T. Kjærgaard, S. Reine, and J. Jakowski, Phys. Chem. Chem. Phys. 14, 15706 (2012).

${ }^{30}$ K. Kristensen, T. Kjærgaard, I.-M. Høyvik, P. Ettenhuber, P. Jørgensen, B. Jansík, S. Reine, and J. Jakowski, Mol. Phys. 111, 1196 (2013).

${ }^{31}$ J. J. Eriksen, P. Baudin, P. Ettenhuber, K. Kristensen, T. Kjærgaard, and P. Jørgensen, J. Chem. Theory Comput. 11, 2984 (2015).

${ }^{32}$ P. Baudin, P. Ettenhuber, S. Reine, K. Kristensen, and T. Kjærgaard, J. Chem. Phys. 144, 054102 (2016).

${ }^{33}$ I.-M. Høyvik, K. Kristensen, T. Kjærgaard, and P. Jørgensen, Theor. Chem. Acc. 133(1), 1417 (2013).

${ }^{34} \mathrm{M}$. Ziołkowski, V. Weijo, P. Jørgensen, and J. Olsen, J. Chem. Phys. 128, 204105 (2008).

${ }^{35}$ P. Ettenhuber and P. Jørgensen, J. Chem. Theory Comput. 11, 1518 (2015).

${ }^{36}$ T. H. Dunning, Jr., J. Chem. Phys. 90, 1007 (1989).

${ }^{37}$ M. Ziółkowski, B. Jansík, P. Jørgensen, and J. Olsen, J. Chem. Phys. 131, 124112 (2009)

${ }^{38}$ I.-M. Høyvik, B. Jansík, and P. Jørgensen, J. Chem. Theory Comput. 8, 3137 (2012). 
${ }^{39}$ See supplementary material at http://dx.doi.org/10.1063/1.4947019 for molecular geometries and the correlation energy of the systems presented in the result section.

${ }^{40}$ LSDALTON, a linear scaling molecular electronic structure program, Release Dalton2016, see http://daltonprogram.org (2016).

${ }^{41}$ K. Aidas, C. Angeli, K. L. Bak, V. Bakken, R. Bast, L. Boman, O. Christiansen, R. Cimiraglia, S. Coriani, P. Dahle, E. K. Dalskov, U. Ekström, T. Enevoldsen, J. J. Eriksen, P. Ettenhuber, B. Fernández, L. Ferrighi, H. Fliegl, L. Frediani, K. Hald, A. Halkier, C. Hättig, H. Heiberg, T. Helgaker, A. C. Hennum, H. Hettema, E. Hjertenæs, S. Høst, I.-M. Høyvik, M. F. Iozzi, B. Jansík, H. J. A. Jensen, D. Jonsson, P. Jørgensen, J. Kauczor, S. Kirpekar, T.
Kjærgaard, W. Klopper, S. Knecht, R. Kobayashi, H. Koch, J. Kongsted, A. Krapp, K. Kristensen, A. Ligabue, O. B. Lutnæs, J. I. Melo, K. V. Mikkelsen, R. H. Myhre, C. Neiss, C. B. Nielsen, P. Norman, J. Olsen, J. M. H. Olsen, A. Osted, M. J. Packer, F. Pawlowski, T. B. Pedersen, P. F. Provasi, S. Reine, Z. Rinkevicius, T. A. Ruden, K. Ruud, V. V. Rybkin, P. Sałek, C. C. M. Samson, A. S. de Merás, T. Saue, S. P. A. Sauer, B. Schimmelpfennig, K. Sneskov, A. H. Steindal, K. O. Sylvester-Hvid, P. R. Taylor, A. M. Teale, E. I. Tellgren, D. P. Tew, A. J. Thorvaldsen, L. Thøgersen, O. Vahtras, M. A. Watson, D. J. D. Wilson, M. Ziolkowski, and H. Ågren, Wiley Interdiscip. Rev.: Comput. Mol. Sci. 4, 269 (2013).

${ }^{42}$ J. W. Boughton and P. Pulay, J. Comput. Chem. 14, 736 (1993). 